\title{
Pricing Routines and Industrial Dynamics
}

\author{
Isabel Almudi ${ }^{1}$, Francisco Fatas-Villafranca , Jesus Palacio , Julio Sanchez-Choliz \\ University of Zaragoza. Gran Via 2-4, 50005 Zaragoza (Spain).
}

\begin{abstract}
We propose an evolutionary model in which boundedly rational firms compete and learn in a dynamic oligopoly with imperfect information and evolving degrees of market power. Firms in the model set prices according to routines, and try to make profits by capturing market share. The model can be extended to deal with heterogeneous costs and technological advance. The demand side of the market is composed of boundedly rational consumers who are capable of adapting to changing market options. Supply-demand interactions can be represented through a population dynamics model from which prices and market structures emerge. We obtain closed-form and simulation results which we interpret and compare with benchmark results from a standard non-cooperative game (Bertrand). When we compare the results with the Bertrand setting, we find a surprising result. Whereas in the fully rational Bertrand setting, firms either lower prices and erode their extra profits, or try to cooperate in a collusive equilibrium that is detrimental for consumer welfare, in the evolutionary setting firms make substantial profits, compete by adjusting prices, and the dynamics improve consumer welfare. From these results we claim that, instead of treating market power, externalities, and asymmetric information as market failures, we should consider them as essential traits of market competition. We argue that neo-Schumpeterian models incorporate all of these features together, thus leading towards a more realistic price theory for market economies.
\end{abstract}

JEL Codes: B52, C62, C72, L11, L13, D43

Keywords: Evolutionary theory, pricing routines, industrial dynamics, game theory, market failures

${ }^{1}$ Correspondence: I. Almudi. Facultad de Economía y Empresa. Universidad de Zaragoza. C/ Gran Vía, 2-4. 50005 (Zaragoza, SPAIN). ialmudi@unizar.es 


\section{Introduction}

From an empirical point of view, it has become increasingly clear that the standard assumptions of neoclassical economic theory are unnecessarily restrictive for studying market economies (Dosi et al. 2016, Dosi et al. 2017, Nelson 2018). Thus, assuming intertemporal rationality of representative agents, perfect (or even probabilistic) access to endogenously changing knowledge, or industrial change from the perspective of static games or equilibrium models is far from satisfactory. In this respect, over the last four decades a number of theorists have been searching for a new approach to economic analysis that draws on the dynamics of evolutionary models (Nelson and Winter 1982, Dosi et al. 1988, Weibull 1995, Metcalfe 1998, Witt 2003, Dopfer et al. 2005, Fatas-Villafranca et al. 2009, Sandholm 2010). Meanwhile, both evolutionary authors (Nelson 2013) and neoclassical industrial organization theorists (Tirole 1988, Fudenberg and Tirole 1991, Hart and Mas-Colell 2003) have insisted on the challenges we still face when trying to understand price formation and markets in modern economies.

Of course, price formation and market outcomes have been persistent themes in standard economic theory (Arrow and Debreu 1954, Debreu 1959, Arrow and Hahn 1971 from a neo-Walrasian perspective; Friedman 1962 or Stigler 1966 in neo-Marshallian frames; Lucas and Prescott 1971, and Stokey, Lucas and Prescott 1989 within the new classical approach to macroeconomics, just to name the classics). Likewise, price formation has been a recurrent theme in standard game theory ever since von Neumann and Morgenstern 1944, Nash 1950, Shubik 1959 and refinements in Harsanyi and Selten 1988 and Van Damme 1987, up until recent evolutionary games (Weibull 1995, Hofbauer and Sigmund 1998, Sandholm 2010). The interest in exploring price dynamics and changing market structures has also been a distinguishing feature of evolutionary economics (Markey-Towler 2016, Bloch and Metcalfe 2018). Nevertheless, even though advances have been achieved, it is well-known that the search for more realistic explanations of price formation and market dynamics is still an open issue in economic theory (Vives 2001, Gallegati et al. 2017).

In this paper, we aim at contributing to the theory of price formation in one sector (one industry) models from an evolutionary perspective. In the evolutionary model we propose, we incorporate market competition and endogenous price formation within a setting in which boundedly rational firms (Simon 1965) and consumer learning (Witt 2001) play key roles. We consider profit-seeking firms that administer

prices according to alternative mark-up routines and try to capture market share (Kalecki 1971, Winter 
1984). On the other side of the market, we model strategic interactions among a large mass of anonymous consumers (Sandholm 2010). Consumers in the model are characterized by inertia, myopia, and a clear intention to improve their choices through the discovery of cheaper options. Thus, our population of heterogeneous consumers learn and adapt to the market by gradually discovering better prices. In turn, demand transformations are asymmetrically perceived by firms, which endogenously change their prices and receive ever-changing profits and market shares. Firms update their supplies to the perceived evolution of demand, and consumers react accordingly. These demand-supply dynamic feedbacks drive an evolutionary process of market competition, which we represent in a tractable model. The process that we obtain resembles the complex approach to markets put forward by Hayek (1948) to a certain extent; an approach in which market competition was conceived as an ongoing discovery process.

Of course we know that, apart from pricing, there are other dimensions that are crucial in market competition. To this regard, we incorporate (as an extension of the basic tractable setting) a wider version of the model in which cost-heterogeneous firms underlying technological advance compete by holding specific mark-up pricing routines. The complete analysis of the complexities that arise in this extension of the model exceeds the scope of a single paper. Nevertheless, drawing upon the extended version of the model, we point out potential lines of research that may inspire theoretical and empirical works from neoSchumpeterian perspectives.

An important aim of this paper is to vindicate the possibility of approaching evolutionary processes through the analysis of not-too-complicated models. In fact, in spite of the attractive features of the enriched version of the model in Section 5, we would like to emphasize the analytical virtues of the simpler setting in Section 3. For instance, the simple approach allows us to obtain genuinely evolutionary closedform propositions that are directly comparable with their neoclassical counterparts (see Section 4). Likewise, the tractable versions of our model (Sections 3 and 5.1) may be more accessible to nonevolutionary scholars and, at the same time, may serve as a well-understandable platform towards more complicated models that can be used by our neo-Schumpeterian colleagues (Section 5.2).

As an illustration of what we mean by engaging scholars from different streams in the discussion, we analyze our evolutionary model of pricing and industrial dynamics by comparing it with a neoclassical pricing exemplar conveniently parameterized for the occasion. The neoclassical benchmark that we use in this paper is the fully-rational perfect knowledge Bertrand game. We analyze this game in its one-shot version, and in its long-run repeated variant. Once we have compared the results in the simple evolutionary setting with the Bertrand game, then we move towards a comparison with the enriched evolutionary 
version (including the generalized case of " $n$ " firms and such neo-Schumpeterian features as cost heterogeneity and technological advance).

An interesting result in the paper is that, whereas in the Bertrand setting firms either mutually destroy their extra-profits or try to collude in a welfare-eroding state, in the evolutionary setting firms make substantial extra profits and tend to adapt prices to ongoing change, while the market generates higher levels of social welfare. Another interesting result is that the model outcomes are qualitatively robust to variations in the specific versions of the mark-up pricing rules and in the number of firms. The robustness of the results and the outcomes of the comparative exercises lead us to argue that, perhaps, instead of treating (as is usual in standard economics) market power, asymmetric information, uncertainty, and externalities as market failures, we should tackle them instead, treating them as essential traits driving markets in the real world. We believe that this comparative analytical approach enriches our understanding of price formation and market processes. Likewise, we consider that the effort we have made to generalize the analysis in a step by step manner widens the potential paths for future theoretical and empirical works.

The paper is organized as follows: in Section 2, we present the basic setting of our evolutionary model for a market process with endogenously changing demand, supply, and pricing. In Section 3, we carry out the dynamic analysis of the model for the cases of dynamic duopolies and oligopolies with constant unit costs. As a benchmark against which we can compare the positive and normative results of the evolutionary model, we then analyze a version of the Bertrand non-cooperative game in Section 4. We discuss the Bertrand model in a one-shot variant, and in a repeated long-run version. We find very different implications when we compare the Bertrand results with those of the basic evolutionary model. Then, in Section 5, we propose an extension of the evolutionary model that considers cost-heterogeneous firms underlying technological advance. We also consider the general case of more than three firms. We compare the new results in the extended version with those obtained in previous sections. Finally, in Section 6, we synthesize our conclusions and propose future research.

\section{An evolutionary model}

\subsection{Basic setting and supply-side assumptions}

We consider an industry composed of $n$ firms $(i=1,2, \ldots, n)$ that offer (almost) perfect substitute varieties of a good or service. Firms compete in prices, with the price of each firm being $p_{i}(t)$. Consumers are boundedly-rational, imperfectly informed, and myopic (Sandholm 2010), and behave according to 
certain inertias in consumption (Nelson 2013), that is to say, they are not revising (in fact they cannot revise) the relative price of their firm provider in comparison with those of the rivals all the time. Having said that, consumers learn from the market, adapt to the new landscape, and can gradually revise their consumption options by looking for better prices.

We assume that, at any time $t$, there is a mass of consumers distributed among a finite number $(n)$ of firms, and thus paying the corresponding firm price and buying the corresponding product. We assume that the size of the industry is constant $Q>0$, and the initial distribution of market shares (initial distribution of the consumers' mass) among the $n$ firms is: $\left\{s_{1}(0), \ldots, s_{n}(0)\right\}$. We assume that each consumer buys one unit of product at any time at the corresponding price $p_{i}(t)$ fixed by $i$-firm at $t$. We also consider that consumers need to buy the good continuously (it is a final consumption good such as bread, food, clothes, mineral water, or specific consumption services).

Regarding supply, we assume that the firms produce according to a production function in which the only input is labor, $Q_{i}(t)=L_{i}(t)$, with productivity being common among firms, and constant and equal to one (we will relax this assumption later on). For the sake of simplicity, we exclude the problems related to capital and we assume that labor is paid a salary $w$ (which we use as a numerairy) and we set the wage $w=1$. For this reason, and given that we are not considering productivity differentials in this basic setting, the unit cost of production of all the firms will be $c=w=1$ (we extend the model for the case of heterogeneous innovative firms in Section 5). The preceding assumptions allow us to define the profits of each firm $i=1,2, \ldots, n$ as:

$$
\begin{aligned}
& B_{i}(t)=\left(p_{i}(t)-1\right) s_{i}(t) Q \text {, and the rate of normalized profitability: } \\
& \qquad \pi_{i}(t)=\left(p_{i}(t)-1\right) s_{i}(t), \quad i=1, \ldots, n
\end{aligned}
$$

We assume that firms are boundedly-rational (profit-seekers instead of profit-maximizers) and they fix their prices. Of course, looking at (1) we see that firms have an incentive to fix high prices and increase profitability. Nevertheless, those firms with higher prices may lose market share, and this effect reduces profits (see (1)). Thus, each firm faces a specific trade-off between increasing prices (to raise profits) or lowering prices to maintain or gain market share (which also raises profits). This trade-off is a crucial permanent concern that influences choice from our boundedly-rational firms.

What is crucial in our evolutionary market is that, since the structure of demand will be endogenously changing in an unpredictable way depending on prices, and firms cannot know what their rivals' behaviors or consumers' reactions will be, each organization must administer its own price in real time. We consider that firms set prices according to specific routines that endogenously depend on the evolving market 
shares. Following Bloch and Metcalfe (2018), we propose mark-up pricing routines that adapt to each firm's demand and to considering the firm unit cost.

More precisely, we assume that firms adaptively vary prices by applying a mark-up on the unit cost $\mathrm{c}=\mathrm{w}=1$ with the mark-up being dependent on the changing market shares. We use the following plausible routines, which are compatible with the theory of mark-up pricing in the literature (Bloch and Metcalfe 2018, Almudi et al. 2012, 2013, Winter 1984, Kalecki 1971):

$$
\begin{aligned}
& \hat{p}_{i}(t)=\frac{\eta_{i} c}{\eta_{i}-s_{i}(t)}, \quad \eta_{i}>1, \quad i=1, \ldots, n . \\
& \tilde{p}_{i}(t)=\left(1+\alpha_{i} s_{i}(t)\right) c, \quad \alpha_{i}>0, \quad i=1, \ldots, n .
\end{aligned}
$$

Since we consider the unit cost $c$ to be equal to one, both expressions are simplified as follows:

$$
\begin{aligned}
& \hat{p}_{i}(t)=\frac{\eta_{i}}{\eta_{i}-s_{i}(t)}, \quad \eta_{i}>1, \quad i=1, \ldots, n . \\
& \tilde{p}_{i}(t)=1+\alpha_{i} s_{i}(t), \quad \alpha_{i}>0, \quad i=1, \ldots, n .
\end{aligned}
$$

Note also that both pricing-rules ${ }^{2}$ capture the idea that, the higher the market share of each firm $i$, the more solid the firm feels in the market, and the higher the margin it is going to charge (thus trying to increase normalized profitability). On the other hand, as firms perceive that they get lower market shares, they react by reducing prices. Note that both expressions (4) and (5) guarantee positive profits; that is to say, the firms' competition to dominate the market and gain increasing profits depends on capturing market share. As we will see below, our boundedly-rational consumers learn and engender an everchanging endogenous distribution of market shares, which drive the unpredictable evolution of demand. Regarding the parameters in the pricing routines (2) to (5), those firms with low values of $\eta_{i}$ or high values of $\alpha_{i}$ are more reactive to changes in market shares. There is a clear relationship between the underlying organizational determinants of these parameters in our setting, organizational inertias, and the concept of firm routines in Nelson and Winter 1982; and Winter 1971.

Finally, we assume that once the firms fix prices - according to (4), (5) - then their production levels and supplies adapt to the reaction of the distribution of market shares (see below), so that, once firms have set and announced their prices, the consumption game we establish below will determine the dynamics of $s_{\mathrm{i}}(t)$ and, consequently, the necessary production levels of each firm, their profits, the endogenous evolution of prices, and the Herfindahl index. Of course, as demand changes, the firms adapt their prices and then demand will react accordingly, thus producing a new change in prices, shares, and profits, and

\footnotetext{
${ }^{2}$ Although, from an economic point of view, both routines are different, when analyzed mathematically we can see that the routine of equation (3) is the development of the first order Taylor series expansion (until the first derivative) of the routine of equation (2) taking $\alpha_{i}=1 / \eta_{i}$ and, thus, the stability of the system will be the same whichever price routine is followed.
} 
so on.

As the sum of market shares is always equal to one, one of the shares can be obtained from the dynamics of the remaining ones, $s_{n}(t)=1-s_{1}(t)-s_{2}(t)-\ldots-s_{n-1}(t)$. Therefore, we can formally work in the (n-1) dimensional simplex. Finally, we shall suppose that the supply of labor is infinitely elastic so that employment is reallocated flexibly between firms in agreement with the needs of production dictated by the demand of each firm. In Fatas-Villafranca et al. (2009) we indicate ways to relax this assumption in an evolutionary computational model.

\subsection{Demand-side assumptions and market interactions}

We will state the assumptions underlying demand and market interactions in the model. As we are working with a price-setting model, which influences demand, and then, subsequently, demand reacts and drives the re-arrangement of prices according to (4) or (5), and everything occurs within a sector with a constant size, the demand and demand variation rates for each firm will be given by the dynamics of market shares $s_{i}(t)$. Here is where we will introduce the fundamental assumptions underlying consumer behavior. We assume that there is a constant large mass of consumers-equal to the size of the market $(Q>0)$ distributed in their purchasing decisions amongst the $i=1, \ldots, n$ firms that exist in the industry. At any time $t$, the proportion of consumers demanding the product variety of firm $i$ at price $p_{i}(t)$ set by the firm is $s_{i}(t)$.

We assume that the consumption of the good produced in this industry does not saturate the budgetary restrictions of consumers who, besides their salaries, may also have other incomes or may spend in other markets. Thus, we rule out income effects and general equilibrium effects.

The $n$ firms in the market (with different prices) represent, from the consumers' point of view, the $n$ alternative buying strategies. In our evolutionary model, instead of assuming that all consumers carefully observe the whole range of options, place them in order according to prices, and then buy the cheapest one, we consider that, given an initial distribution of choices (market shares) $\left(s_{\mathrm{i}}(0)\right), i=1, \ldots, n$, consumers will revise (or may revise) their strategies (consumption option) from time to time, and that they do this in an environment of imperfect information. More precisely, we consider that consumers interact randomly with other consumers who have distinct options, and they communicate and share market information that eventually leads to behavioral changes as the market plays out dynamically. Formally, we assume that consumers aim to buy at lower prices, and they gradually discover information and decide to revise their consumption options. For clarity, we assume that the consumers' payoffs for 
each strategy/firm (consumption option) are given by:

$$
u_{i}(t)=-p_{i}(t), \quad i=1, \ldots, n .
$$

Equation (6) just captures the idea that consumers wish to buy products as cheaply as possible. Hence, we propose that the boundedly rational consumers will gradually try to discover cheaper varieties of the good, but always depart from specific initial conditions and deploy their action in a path-dependent way. This assumption, which is in agreement with a revision protocol we shall now present, will drive the endogenous evolution of demand and the bidirectional relations linking unpredictable demand change and firms’ pricing.

Revision protocol: We assume a pairwise random matching mechanism between consumers with the probability of consumer meetings in the market being proportional to the firm's market shares (i.e. to the proportions of consumers buying $i$ or $j$ at any time), so that, the probability that those consumers who are consuming varieties $i$ and $j$ at any time (with $i, j=1, \ldots n$ ) may meet and communicate is proportional to the size of the groups of consumers, $\beta s_{i}(t) s_{j}(t)$, with $0<\beta<1$ being a parameter indicating the easiness or difficulty of the meetings coming about. Parameter $\beta$ measures the intensity of competition in the sector. To complete the revision protocol we must set a switching-function. We state that the flow of consumers, after meeting randomly, may revise their consumption options (the variety of the good they buy, i.e. the firm they buy from) from $j$ to $i$ at $t$, will be denoted by $f_{i j}(t)$, and we define the switching revision process as follows:

$$
\begin{aligned}
f_{i j}(t)=\max \left\{u_{i}(t)-u_{j}(t) ; 0\right\}= & \max \left\{p_{j}(t)-p_{i}(t) ; 0\right\} . \text { As a consequence of this we have: } \\
& f_{i j}(t)-f_{j i}(t)=p_{j}(t)-p_{i}(t)
\end{aligned}
$$

With this type of revision protocol embedded in the market process of discovery, we assure that the flow of consumers always operates towards the cheaper varieties, as consumers gradually discover and explore the range of prices which, in turn, changes endogenously according to pricing routines (4) or (5). Thus, we have inertial and myopic consumers who, eventually, randomly meet in pairwise contests, communicate in the market, and may decide to migrate from more expensive to cheaper firms. It is crucial to mention that, as consumers gradually leave certain firms by moving to other cheaper firms, and considering that firms adapt their prices following (4) or (5), consumer migrations generate externalities in other consumers (via prices). More precisely, as a certain mass of consumers leave from an expensive firm, they force this firm to lower its price. Likewise, as the mass of consumers arrives at a cheaper firm, they push the low price of this firm upwards. As we will see below, these migrations with externalities are essential to understanding the equilibrating process that drives the market in the model. Having said that, 
and considering pairwise random matchings, it is clear that, in continuous time, the evolution of each firm i's market share-i.e. the evolution of the proportion of consumers buying from firm $i$ (players playing strategy $i$ within this population game)— will be:

$$
\begin{aligned}
& \quad \dot{s}_{i}=\sum_{j=1}^{n} \beta s_{i}(t) s_{j}(t)\left(f_{i j}(t)-f_{j i}(t)\right)=\sum_{j=1}^{n} \beta s_{i}(t) s_{j}(t)\left(p_{j}(t)-p_{i}(t)\right)= \\
& =\beta s_{i}(t) \sum_{j=1}^{n} s_{j}(t)\left(p_{j}(t)-p_{i}(t)\right) .
\end{aligned}
$$

Defining the average price as $\bar{p}(t)=\sum_{i=1}^{n} s_{i}(t) p_{i}(t)$ we see that the evolution of the market will be:

$$
\dot{s}_{i}=\beta s_{i}(t)\left(\bar{p}(t)-p_{i}(t)\right), \quad i=1, \ldots, n .
$$

with $p_{i}(t)$ being given by the pricing routines of equations (4) and/or (5) as necessary. Notice that equations (1), (4), (5), and (9) fully represent an evolutionary market with endogenously changing prices, market shares, concentration index, profits, and levels of consumer satisfaction (see the payoffs in (6)). The replicator dynamics in (9) are the core representation of our market. The prices (the fitness levels) change endogenously in a way that makes the properties that may emerge from (9) not obvious at all. In order to study these dynamics, we devote subsection 3.1 to carrying out, first, the dynamic analysis for the duopoly case. Then, in subsection 3.2, we will focus on a three-firm setting as a representation of the dynamic oligopoly case. Later on, in Section 4, we will explore a Bertrand-pricing (one-sector) game as a neoclassical benchmark against which we will be able to compare our evolutionary results. Finally, in Section 5, we will extend the model to the case of cost-heterogeneous firms bringing about technological advance. How to extend the model for more than three firms will also be discussed.

\section{Dynamic Analysis}

\subsection{Duopoly}

In order to study the model, first we carry out a stability analysis for the evolution of the dynamic-duopoly case (we fix $n=2$ in our evolutionary model). We first detect the resting points, characterize their stability, and interpret the overall dynamics of the market. We obtain results regarding market concentration, profits and consumer welfare. In the $n=2$ case, since the market share of the second firm, $s_{2}(t)$, can be written as a function of the first firm's share, $s_{2}(t)=1-s_{1}(t)$, the market will be driven by just one equation:

$$
\dot{s}_{1}(t)=\beta s_{1}(t)\left(1-s_{1}(t)\right)\left(p_{2}(t)-p_{1}(t)\right) \text {. }
$$

\subsubsection{Stability}

Equation (10) clearly shows that the firm with a lower price at any time tends to gain market share. As we 
have said, firms administer their prices (equations (4) and (5)) according to the perceived evolution of demand (market share). Therefore, apart from equation (10) driving market competition, and equation (1) in which we see profit evolution, we need to incorporate the pricing equations (4) and (5) to study the stability and dynamics of the market. We shall study the stability of the replicator dynamics (10), taking first the pricing routines of equation (4), and then those of equation (5). Thus, from (4) and (10), we obtain:

$$
\dot{s}_{1}(t)=\hat{f}\left(s_{1}\right)=\beta s_{1}(t)\left(1-s_{1}(t)\right)\left(\frac{\eta_{2}}{\eta_{2}-1+s_{1}(t)}-\frac{\eta_{1}}{\eta_{1}-s_{1}(t)}\right)
$$

This equation (11) has three critical points, $s_{1}=0, s_{1}=1$ and $s_{1}=\frac{\eta_{1}}{\eta_{1}+\eta_{2}}$. The critical points $s_{1}=$ $0, s_{1}=1$ represent situations of monopoly, while $s_{1}=\frac{\eta_{1}}{\eta_{1}+\eta_{2}}$, with $s_{2}=\frac{\eta_{2}}{\eta_{1}+\eta_{2}}$ represents a situation of duopoly with the two firms sharing the market depending on their pricing routines. More precisely, if we rewrite (4) as $\hat{p}_{i}(t)=\frac{1}{1-s_{i} / \eta_{i}}, \quad \eta_{i}>1, \quad i=1,2$, we see that $1 / \eta_{i}$ can be interpreted as the propensity to change price when adapting prices to variations in market share. Thus, parameter $\eta_{i}$ could be seen as the inertia of firm $i$ when re-setting prices adapting to changing demand. The higher the value of $\eta_{i}$, the lower the price-reactivity of firm $i$ to its changing market share. It is interesting that, at the critical point at which $s_{1}=\frac{\eta_{1}}{\eta_{1}+\eta_{2}}, s_{2}=\frac{\eta_{2}}{\eta_{1}+\eta_{2}}$ (both firms end up sharing the market), the more inertial firm (the one less volatile in adapting prices to demand) is the one with a higher market share.

We shall check now the stability of critical points. To do this, we evaluate the equation in $\bar{s}_{0} \in\left(0, \frac{\eta_{1}}{\eta_{1}+\eta_{2}}\right)$ and in $\hat{s}_{0} \in\left(\frac{\eta_{1}}{\eta_{1}+\eta_{2}}, 1\right)$. We see that $\hat{f}\left(\bar{s}_{0}\right)>0$ and $\hat{f}\left(\hat{s}_{0}\right)<0$, and, thus, points $s_{1}=0$ and $s_{1}=1$ are unstable, while point $s_{1}=\frac{\eta_{1}}{\eta_{1}+\eta_{2}}$ is stable. This can be seen in a graphic representation for stability in Figure 1. This analysis tells us that any route $s_{1}^{*}(t)$ with $s_{1}^{*}(0) \neq 1$ and $s_{1}^{*}(0) \neq 0$ will tend, when $t \rightarrow$ $\infty$, to $\lim _{t \rightarrow \infty} s_{1}(t)=\frac{\eta_{1}}{\eta_{1}+\eta_{2}}$. Therefore, the third critical point at which both firms end up with identical prices and sharing the market $s_{1}=\frac{\eta_{1}}{\eta_{1}+\eta_{2}}, s_{2}=\frac{\eta_{2}}{\eta_{1}+\eta_{2}}$, is the stable limit situation of the market processwhen firms set prices following (4) (see Figure 1). 


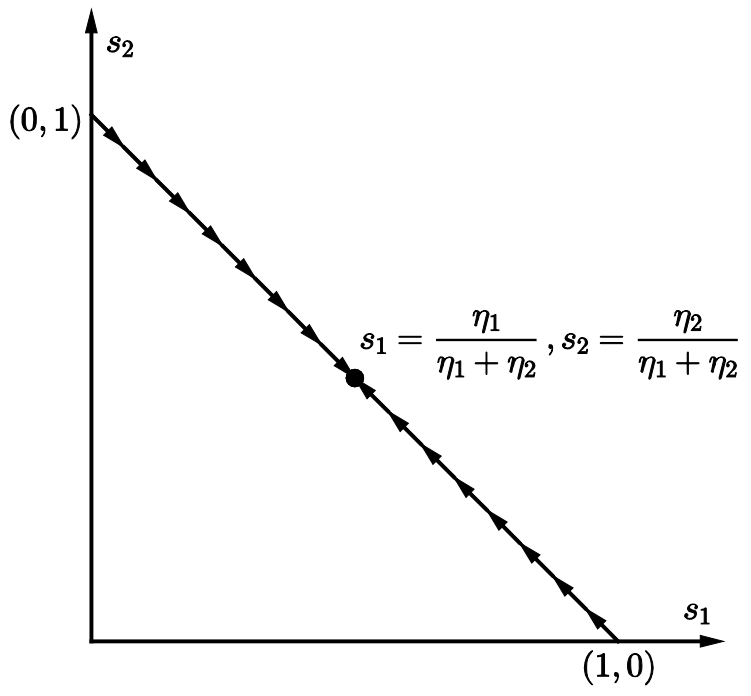

Figure 1. Stability for differential equation (11).

If we take the pricing routine of equation (5) now, the replicator dynamics are:

$$
\dot{s}_{1}(t)=\tilde{f}\left(s_{1}\right)=\beta s_{1}(t)\left(1-s_{1}(t)\right)\left(\alpha_{2}\left(1-s_{1}(t)\right)-\alpha_{1} s_{1}\right)
$$

The critical points of this equation are $s_{1}=0, s_{1}=1$ and $s_{1}=\frac{\alpha_{2}}{\alpha_{1}+\alpha_{2}}$. We proceed in a similar way to the previous case and we evaluate the function for $\bar{s}_{0} \in\left(0, \frac{\alpha_{2}}{\alpha_{1}+\alpha_{2}}\right)$ and for $\hat{s}_{0} \in\left(\frac{\alpha_{2}}{\alpha_{1}+\alpha_{2}}, 1\right)$. We can see that $\tilde{f}\left(\bar{s}_{0}\right)>0$ and $\tilde{f}\left(\hat{s}_{0}\right)<0$, so points $s_{1}=0$ and $s_{1}=1$ are unstable while point $s_{1}=\frac{\alpha_{2}}{\alpha_{1}+\alpha_{2}}$ is stable. Here again we find that the firm with a less reactive (more inertial) pricing routine is the one that ends up controlling a higher share of the market. The graphical representation of stability is analogous to that of Figure 1. Therefore, for both pricing routines, we find that the interior critical stationary state of the market is the asymptotically stable state. The stabilities of the results for both routines are similar as we could have supposed since we have already proved that one of the routines is the first-order Taylor expansion of the other.

\subsubsection{Equilibria, profitability, consumers’ payoff, and market structure}

As a consequence of the results in 3.1.1, we are going to focus now on the asymptotically stable state, and we shall obtain and interpret further characteristics of this market situation. We have seen that, for the pricing routines of equation (4) the equilibrium is reached at $\hat{s}^{*}=\left(\frac{\eta_{1}}{\eta_{1}+\eta_{2}}, \frac{\eta_{2}}{\eta_{1}+\eta_{2}}\right)$ and for those of equation (5), it is at $\tilde{S}^{*}=\left(\frac{\alpha_{2}}{\alpha_{1}+\alpha_{2}}, \frac{\alpha_{1}}{\alpha_{1}+\alpha_{2}}\right)$. Starting out from these results, we can see what the normalized 
profitability is for firms, the consumers’ pay-offs, and the Herfindahl index.

\section{Normalized profitability}

Remember that normalized profitability is given by equation (1) such that:

$$
\begin{aligned}
& \hat{\pi}_{i}^{*}=\left(\hat{p}_{i}^{*}-1\right) \hat{s}_{i}^{*}=\left(\frac{\eta_{i}}{\eta_{i}-\hat{s}_{i}^{*}}-1\right) \hat{s}_{i}^{*}=\frac{\hat{s}_{i}^{* 2}}{\eta_{i}-\hat{s}_{i}^{*}}=\frac{\frac{\eta_{i}^{2}}{\left(\eta_{i}+\eta_{j}\right)^{2}}}{\eta_{i}-\frac{\eta_{i}}{\eta_{i}+\eta_{j}}}= \\
& =\frac{\eta_{i}}{\left(\eta_{i}+\eta_{j}\right)\left(\eta_{i}+\eta_{j}-1\right)^{\prime}} \\
& \tilde{\pi}_{i}^{*}=\left(\tilde{p}_{i}^{*}-1\right) \tilde{s}_{i}^{*}=\alpha_{i} \tilde{s}_{i}^{* 2}=\frac{\alpha_{i} \alpha_{j}^{2}}{\left(\alpha_{i}+\alpha_{j}\right)^{2}} .
\end{aligned}
$$

As we see in (13) and (14), in the emergent stable situation of the market, both firms make extra profits for both pricing routines. Both firms end up fixing equal prices in the endogenously emergent limit market state, and both firms have similar unit costs (equal to 1). Then, we see from equations (13) and (14) that the firm that ends up being more profitable (although both of them make positive extra profits) is the one with higher-limit market share; the most profitable firm in the limit-state of the market will be the one that is less sensitive to changing demand in the administration of its pricing routine.

Of course, full-rigidity is not a realistic case since price fine tuning is the crucial mechanism underlying the transient dynamic process out of equilibrium. But it remains clear that being sufficiently inertial (not changing in a highly-reactive way) is a plus in the final equilibrium state.

\section{Consumer payoffs}

In our model, notice that the payoff for consumers is given by equation (6). Then, in the limit market state of the dynamic duopoly, the payoff gained by consumers buying from either firm 1 or firm 2 must be the same, $u_{1}^{*}=u_{2}^{*}$. This is indeed the case, since we have already seen that prices in the asymptotically stable market state tend to be identical, otherwise, consumers would gradually find (according to the learning revision protocol) a cheaper option and the shares would change. This is something that does not happen at the stable resting point, although it is continuously happening in the path-dependent paths converging towards the stable state. The stable state is an emergent limit property of our evolving industry, in which consumers have exhausted the search opportunities in the market, firms have set identical prices, and both groups of customers earn a payoff $u_{1}^{*}=u_{2}^{*}=u^{*}=-p^{*}$, with $p^{*}$ denoting the identical firm-price in equilibrium (adding a hat or tilde according to the price routine we are considering). Thus, we have: 


$$
\begin{aligned}
& \hat{u}^{*}=-\hat{p}^{*}=-\frac{\eta_{i}}{\eta_{i}-\hat{s}_{i}^{*}}=\frac{-\eta_{i}\left(\eta_{i}+\eta_{j}\right)}{\eta_{i}\left(\eta_{i}+\eta_{j}\right)-\eta_{i}}=\frac{\eta_{i}+\eta_{j}}{1-\eta_{i}-\eta_{j}} \\
& \tilde{u}^{*}=-\tilde{p}^{*}=-1-\alpha_{i} \tilde{s}_{i}^{*}=-1-\alpha_{i} \frac{\alpha_{j}}{\alpha_{i}+\alpha_{j}}=\frac{-\alpha_{i}-\alpha_{j}-\alpha_{i} \alpha_{j}}{\alpha_{i}+\alpha_{j}} .
\end{aligned}
$$

It is worth noticing that the criteria for increasing consumer-payoff (low prices) goes in the opposite direction to the criteria for increasing firm profits (high prices). Setting out from initial and parametric conditions, the market process operates through the equilibrating role of consumer migration looking for low prices, externalities of consumer migrations upon other consumers, new migrations leading to firm prices being updated, new prices signaling new opportunities, and unchaining new consumer migrations. This process tends towards a limit-state in which both firms make extra profits. They do so by fixing an (identical) level of prices.

\section{Herfindahl index}

Finally, in the evolutionary duopoly case, the Herfindahl index in the equilibrium is given by $H=s_{1}^{* 2}+$ $s_{2}^{* 2}$. Thus, substituting different $s^{*}$ according to the price routines:

$$
\begin{gathered}
\widehat{H}=\hat{s}_{1}^{* 2}+\hat{s}_{2}^{* 2}=\left(\frac{\eta_{1}}{\eta_{1}+\eta_{2}}\right)^{2}+\left(\frac{\eta_{2}}{\eta_{1}+\eta_{2}}\right)^{2}=\frac{\eta_{1}^{2}+\eta_{2}^{2}}{\left(\eta_{1}+\eta_{2}\right)^{2}} \\
\widetilde{H}=\tilde{s}_{1}^{2}+\tilde{s}_{2}^{2}=\left(\frac{\alpha_{2}}{\alpha_{1}+\alpha_{2}}\right)^{2}+\left(\frac{\alpha_{1}}{\alpha_{1}+\alpha_{2}}\right)^{2}=\frac{\alpha_{1}^{2}+\alpha_{2}^{2}}{\left(\alpha_{1}+\alpha_{2}\right)^{2}} .
\end{gathered}
$$

The Herfindahl index is at a minimum when it is equal to $1 / n$, in our case $1 / 2$, which is produced when both firms have the same market share. This minimum value is reached if and only if firms have the same price-routine parameters, that is, $\eta_{1}=\eta_{2}$ for the first price routine and $\alpha_{1}=\alpha_{2}$ for the second one. In an evolutionary world this is an extremely rare event. Behavioral diversity and heterogeneous organizational conditions are the regular norm, so we should not expect the appearance of perfectly symmetric markets to be frequent. We will dig deeper into the role of firm heterogeneity in Section 5. For the time being, let us remark that the model dynamics rule out the possibility of $H$ being one (monopoly, its maximum value) since we have proved that those are unstable states of the market.

\subsection{Oligopoly}

We shall focus now on the case of three firms competing in the sector. This is closer to a typical oligopoly case. The results reinforce the generality of the properties obtained above. As with the case of the duopoly, 
analyzing the market share of two of the firms will be sufficient, as the third firm's share will be an expression of the other two firms, $s_{3}(t)=1-s_{1}(t)-s_{2}(t)$. Hence the market dynamics in this $n=3$ oligopoly case will be reduced to a system of two ordinary differential equations:

$$
\begin{aligned}
& \dot{s}_{1}(t)=\beta s_{1}(t)\left[s_{2}(t)\left(p_{2}(t)-p_{1}(t)\right)+\left(1-s_{1}(t)-s_{2}(t)\right)\left(p_{3}(t)-p_{1}(t)\right)\right] ; \\
& \dot{s}_{2}(t)=\beta s_{2}(t)\left[s_{1}(t)\left(p_{1}(t)-p_{2}(t)\right)+\left(1-s_{1}(t)-s_{2}(t)\right)\left(p_{3}(t)-p_{2}(t)\right)\right] .
\end{aligned}
$$

As before, the final dynamic system we obtain will depend on the pricing routines chosen. For the price routine of equation (4) we see:

$$
\begin{aligned}
\dot{s}_{1}(t)=\hat{f}_{1}\left(s_{1}, s_{2}\right)= & \beta s_{1}(t)\left[s_{2}(t)\left(\frac{\eta_{2}}{\eta_{2}-s_{2}(t)}-\frac{\eta_{1}}{\eta_{1}-s_{1}(t)}\right)+\right. \\
& \left.+\left(1-s_{1}(t)-s_{2}(t)\right)\left(\frac{\eta_{3}}{\eta_{3}-1+s_{1}(t)+s_{2}(t)}-\frac{\eta_{1}}{\eta_{1}-s_{1}(t)}\right)\right] ; \\
\dot{s}_{2}(t)=\hat{f}_{2}\left(s_{1}, s_{2}\right)= & \beta s_{2}(t)\left[s_{1}(t)\left(\frac{\eta_{1}}{\eta_{1}-s_{1}(t)}-\frac{\eta_{2}}{\eta_{2}-s_{2}(t)}\right)+\right. \\
& \left.+\left(1-s_{1}(t)-s_{2}(t)\right)\left(\frac{\eta_{3}}{\eta_{3}-1+s_{1}(t)+s_{2}(t)}-\frac{\eta_{2}}{\eta_{2}-s_{2}(t)}\right)\right]
\end{aligned}
$$

and for the price routine of equation (5) we see:

$$
\begin{aligned}
\dot{s}_{1}(t)=\tilde{f}_{1}\left(s_{1}, s_{2}\right)= & \beta s_{1}(t)\left[s_{2}(t)\left(\alpha_{2} s_{2}(t)-\alpha_{1} s_{1}(t)\right)+\right. \\
& \left.+\left(1-s_{1}(t)-s_{2}(t)\right)\left(\alpha_{3}\left(1-s_{1}(t)-s_{2}(t)\right)-\alpha_{1} s_{1}(t)\right)\right] ; \\
\dot{s}_{2}(t)=\tilde{f}_{2}\left(s_{1}, s_{2}\right)= & \beta s_{2}(t)\left[s_{1}(t)\left(\alpha_{1} s_{1}(t)-\alpha_{2} s_{2}(t)\right)+\right. \\
& \left.+\left(1-s_{1}(t)-s_{2}(t)\right)\left(\alpha_{3}\left(1-s_{1}(t)-s_{2}(t)\right)-\alpha_{2} s_{2}(t)\right)\right]
\end{aligned}
$$

As we have seen in 3.1, as certain masses of consumers learn and revise their options in continuous time, they can operate as stabilizers of the market. These consumer flows induce expensive firms to lower their prices and allow for cheaper firms to increase their prices. This equilibrating process drives the price vector in real time, which results from market self-organization and does not come from any fictitious auctioneer operating in the vacuum. It is the outcome of step-by-step learning and trading, and endogenous adaptations of consumers and firms in continuous time, which underlie the ongoing evolutionary process.

\subsubsection{Stability}

We begin the exploration by analyzing the stability characteristics of dynamic systems (19) and (20).

For the first dynamic system (19), we obtain the following critical points: $\hat{A}=(0,0), \hat{B}=(0,1), \hat{C}=$ $\left(0, \frac{\eta_{2}}{\eta_{2}+\eta_{3}}\right), \widehat{D}=(1,0), \hat{E}=\left(\frac{\eta_{1}}{\eta_{1}+\eta_{3}}, 0\right), \hat{F}=\left(\frac{\eta_{1}}{\eta_{1}+\eta_{2}}, \frac{\eta_{2}}{\eta_{1}+\eta_{2}}\right)$ and $\hat{G}=\left(\frac{\eta_{1}}{\eta_{1}+\eta_{2}+\eta_{3}}, \frac{\eta_{2}}{\eta_{1}+\eta_{2}+\eta_{3}}\right)$. The points $\hat{A}=(0,0), \hat{B}=(0,1), \widehat{D}=(1,0)$ are alternative monopoly states. Points $\hat{C}=\left(0, \frac{\eta_{2}}{\eta_{2}+\eta_{3}}\right), \hat{E}=$ 
$\left(\frac{\eta_{1}}{\eta_{1}+\eta_{3}}, 0\right), \hat{F}=\left(\frac{\eta_{1}}{\eta_{1}+\eta_{2}}, \frac{\eta_{2}}{\eta_{1}+\eta_{2}}\right)$ are duopolistic states (two firms remain in the market). The point $\hat{G}=$ $\left(\frac{\eta_{1}}{\eta_{1}+\eta_{2}+\eta_{3}}, \frac{\eta_{2}}{\eta_{1}+\eta_{2}+\eta_{3}}\right)$ is a resting point at which the three firms in the oligopoly share the market according to (normally) different limit market shares $s_{\mathrm{i}}=\frac{\eta_{\mathrm{i}}}{\eta_{1}+\eta_{2}+\eta_{3}}, i=1,2,3$. To see the local stability at each point, we calculate the Jacobian matrix of $\hat{f}\left(s_{1}, s_{2}\right)=\left(\hat{f}_{1}\left(s_{1}, s_{2}\right), \hat{f}_{2}\left(s_{1}, s_{2}\right)\right)$, we evaluate it at each point, and we study its eigenvalues and eigenvectors. We achieved the following results:

- $\hat{A}=(0,0)$. The eigenvalues of the Jacobian matrix are equal and positive, $\lambda_{1}=\lambda_{2}=$ $\frac{\beta}{\eta_{3}-1}>0$ and the eigenvectors are $v_{1}=(1,0), v_{2}=(0,1)$. Thus we are faced with an unstable point from which trajectories point out in all directions.

- $\hat{B}=(0,1)$. The eigenvalues of the Jacobian are equal and positive, $\lambda_{1}=\lambda_{2}=\frac{\beta}{\eta_{2}-1}>0$ and the eigenvectors are $v_{1}=(1,0), v_{2}=(0,1)$. This gives an unstable point from which trajectories point out in all directions.

- $\hat{C}=\left(0, \frac{\eta_{2}}{\eta_{2}+\eta_{3}}\right)$. The eigenvalues are $\lambda_{1}=\frac{\beta}{\eta_{2}+\eta_{3}-1}>0$ and $\lambda_{2}=\frac{-\beta\left(\eta_{2}+\eta_{3}\right)}{\left(\eta_{2}+\eta_{3}-1\right)^{2}}<0$. With associated eigenvectors $v_{1}=\left(\frac{-\eta_{2}-\eta_{3}}{\eta_{2}}, 1\right)$ and $v_{2}=(0,1)$. Thus, it is a saddle point the attracting vector of which is $v_{2}$.

- $\widehat{D}=(1,0)$. The eigenvalues of the Jacobian matrix are equal and positive, $\lambda_{1}=\lambda_{2}=$ $\frac{\beta}{\eta_{1}-1}>0$ and the eigenvectors are $v_{1}=(1,0), v_{2}=(0,1)$. Once again, we have an unstable point. Notice that the monopoly situations are three unstable market states.

- $\hat{E}=\left(\frac{\eta_{1}}{\eta_{1}+\eta_{3}}, 0\right)$. The eigenvalues are $\lambda_{1}=\frac{\beta}{\eta_{1}+\eta_{3}-1}>0$ and $\lambda_{2}=\frac{-\beta\left(\eta_{1}+\eta_{3}\right)}{\left(\eta_{1}+\eta_{3}-1\right)^{2}}<0$. With eigenvectors $v_{1}=\left(\frac{-\eta_{1}}{\eta_{1}+\eta_{3}}, 1\right)$ and $v_{2}=(1,0)$. A saddle point the attracting vector of which is $v_{2}$.

- $\hat{F}=\left(\frac{\eta_{1}}{\eta_{1}+\eta_{2}}, \frac{\eta_{2}}{\eta_{1}+\eta_{2}}\right)$. The eigenvalues are $\lambda_{1}=\frac{\beta}{\eta_{1}+\eta_{2}-1}>0$ and $\lambda_{2}=\frac{-\beta\left(\eta_{1}+\eta_{2}\right)}{\left(\eta_{1}+\eta_{2}-1\right)^{2}}<0$. With associated eigenvectors $v_{1}=\left(\frac{\eta_{1}}{\eta_{2}}, 1\right)$ and $v_{2}=(-1,1)$. Thus, it is a saddle point with 
attracting vector $v_{2}$. Notice that the duopoly resting points are unstable saddle points.

- $\hat{G}=\left(\frac{\eta_{1}}{\eta_{1}+\eta_{2}+\eta_{3}}, \frac{\eta_{2}}{\eta_{1}+\eta_{2}+\eta_{3}}\right)$. The eigenvalues of the Jacobian matrix are equal and negative, $\lambda_{1}=\lambda_{2}=\frac{-\beta\left(\eta_{1}+\eta_{2}+\eta_{3}\right)}{\eta_{1}+\eta_{2}+\eta_{3}-1}<0$ and the eigenvectors are $v_{1}=(1,0), v_{2}=(0,1)$, an asymptotically stable point to which trajectories arrive from all directions. Thus, the $\hat{G}$ point at which the three firms survive is the stable point towards which the equilibrating market process in our evolutionary model (with bounded rationality, imperfect information, externalities, evolving market power, pricing and learning) leads the system.

We can graphically depict (for a representative parametric setting) ${ }^{3}$ the dynamics, which we have formally obtained above, in Figure 2. We take as parameter values $\beta=0.5, \eta_{1}=1.1, \eta_{2}=1.4$ and $\eta_{3}=1.2$, in such a way that the critical points in terms of firm market shares are: $\hat{A}=(0,0), \hat{B}=(0,1)$, $\hat{C}=(0,0.538), \widehat{D}=(1,0), \hat{E}=(0.478,0), \hat{F}=(0.44,0.56)$ and $\hat{G}=(0.297,0.378)$.

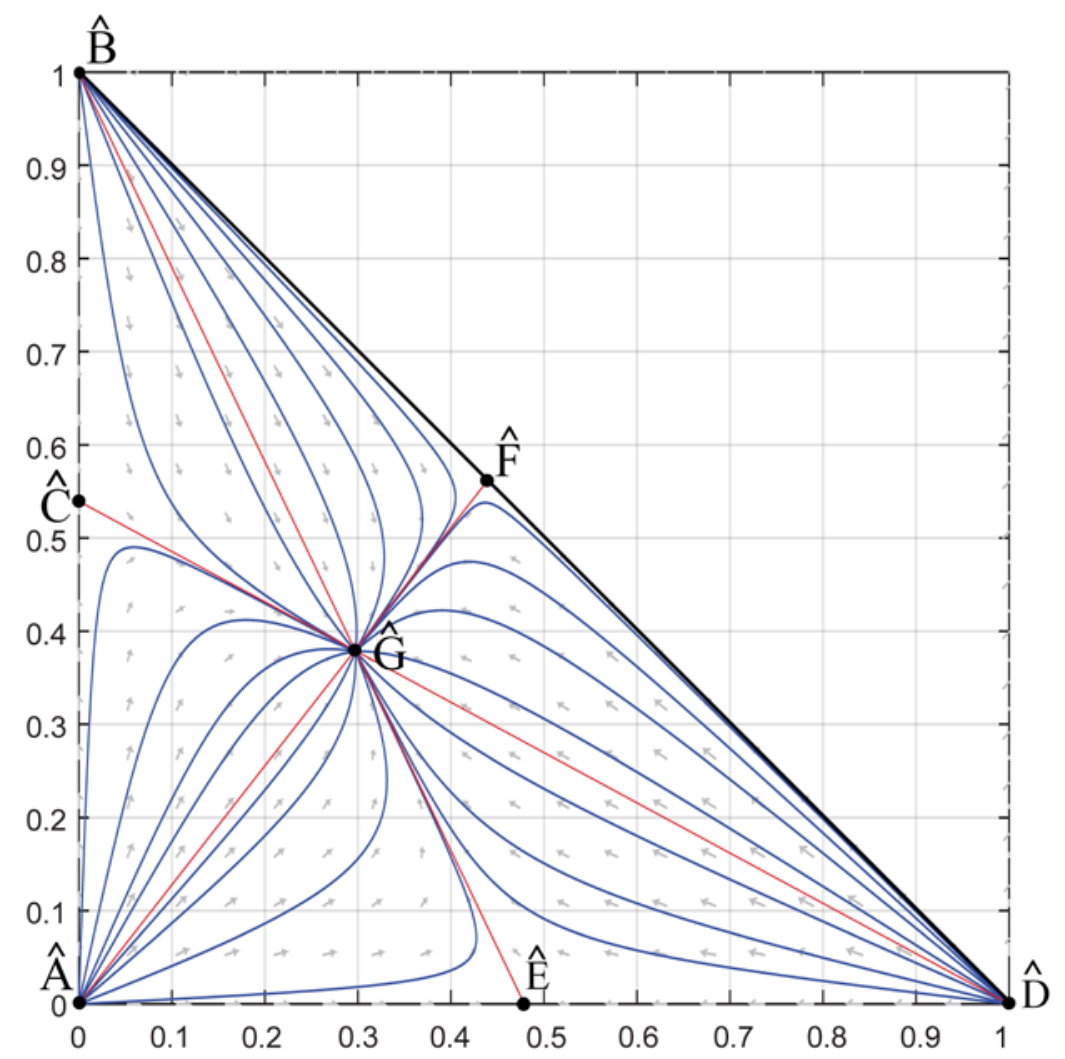

Figure 2. Phase diagram for the replicator dynamics (19). On the horizontal axis, share of firm 1; vertical axis, share of firm 2. Parameter values: $\beta=0.5, \eta_{1}=1.1, \eta_{2}=1.4$ and $\eta_{3}=1.2$. The share of firm 3 is $s_{3}(t)=1-s_{1}(t)-s_{2}(t)$.

\footnotetext{
${ }^{3}$ The graphical representation was carried out with pplane.m software developed by John C. Polking. For more information, see [Polking and Arnold, 2004].
} 
Thus we can see that there is only one stable point, $\hat{G}$. All the trajectories of the system lead towards $\hat{G}$ except those on the boundary of the $n-1$ dimensional simplex; that is, $1 \neq s_{i}(0) \neq 0$ for $i=1,2$ and $s_{1}(0)+s_{2}(0) \neq 1$. Note that, in the case of $s_{1}(0)+s_{2}(0)=1$, we would obtain the same results as for the duopoly case, with $\widehat{F}$ being the stable point of this duopoly. Compare to Figure 1 . We assume in the present oligopoly case that we set out from the interior of the $n$ - 1 simplex so that we can focus attention on $\widehat{G}$ and the dynamics leading to it.

Now, we shall look at the second market system (20) with alternative pricing routines. We obtain the following critical points: $\tilde{A}=(0,0), \tilde{B}=(0,1), \tilde{C}=\left(0, \frac{\alpha_{3}}{\alpha_{2}+\alpha_{3}}\right), \tilde{D}=(1,0), \tilde{E}=\left(\frac{\alpha_{3}}{\alpha_{1}+\alpha_{3}}, 0\right), \tilde{F}=$ $\left(\frac{\alpha_{2}}{\alpha_{1}+\alpha_{2}}, \frac{\alpha_{1}}{\alpha_{1}+\alpha_{2}}\right)$ and finally $\tilde{G}=\left(\frac{\alpha_{2} \alpha_{3}}{\alpha_{1} \alpha_{2}+\alpha_{2} \alpha_{3}+\alpha_{1} \alpha_{3}}, \frac{\alpha_{1} \alpha_{3}}{\alpha_{1} \alpha_{2}+\alpha_{2} \alpha_{3}+\alpha_{1} \alpha_{3}}\right)$.

To check the stability of the points, we shall use the same method as with the previous case.

- $\tilde{A}=(0,0)$. The eigenvalues of the Jacobian matrix are equal and positive, $\lambda_{1}=\lambda_{2}=$ $\beta \alpha_{3}>0$ and the eigenvectors are $v_{1}=(1,0), v_{2}=(0,1)$. We have an unstable point from which trajectories start out in all directions.

- $\tilde{B}=(0,1)$. The eigenvalues of the Jacobian matrix are equal and positive, $\lambda_{1}=\lambda_{2}=$ $\beta \alpha_{2}>0$ and the eigenvectors are $v_{1}=(1,0), v_{2}=(0,1)$. We have an unstable point from which trajectories start out in all directions.

- $\tilde{C}=\left(0, \frac{\alpha_{3}}{\alpha_{2}+\alpha_{3}}\right)$. Eigenvalues are $\lambda_{1}=\frac{\beta \alpha_{2} \alpha_{3}}{\alpha_{2}+\alpha_{3}}>0$ and $\lambda_{2}=\frac{-\beta \alpha_{2} \alpha_{3}}{\left.\alpha_{2}+\alpha_{3}\right)^{2}}<0$. With associated eigenvectors $v_{1}=\left(\frac{\alpha_{2}+\alpha_{3}}{\alpha_{3}},-1\right)$ and $v_{2}=(0,1)$. Thus it is a saddle point the attractor of which is $v_{2}$.

- $\widetilde{D}=(1,0)$. The eigenvalues of the Jacobian are equal and positive, $\lambda_{1}=\lambda_{2}=\beta \alpha_{1}>0$ and the eigenvectors are $v_{1}=(1,0), v_{2}=(0,1)$. We have an unstable point from which trajectories set out in all directions.

- $\tilde{E}=\left(\frac{\alpha_{3}}{\alpha_{1}+\alpha_{3}}, 0\right)$. The eigenvalues are $\lambda_{1}=\frac{\beta \alpha_{1} \alpha_{3}}{\alpha_{1}+\alpha_{3}}>0$ and $\lambda_{2}=\frac{-\beta \alpha_{1} \alpha_{3}}{\alpha_{1}+\alpha_{3}}<0$. With 
associated eigenvectors $v_{1}=\left(\frac{\alpha_{3}}{\alpha_{3}+\alpha_{1}},-1\right)$ and $v_{2}=(1,0)$. A saddle point the attractor of which is $v_{2}$.

- $\tilde{F}=\left(\frac{\alpha_{2}}{\alpha_{1}+\alpha_{2}}, \frac{\alpha_{1}}{\alpha_{1}+\alpha_{2}}\right)$. The eigenvalues are $\lambda_{1}=\frac{\beta \alpha_{1} \alpha_{2}}{\alpha_{1}+\alpha_{2}}>0$ and $\lambda_{2}=\frac{-\beta \alpha_{1} \alpha_{2}}{\alpha_{1}+\alpha_{2}}<0$. With associated eigenvectors $v_{1}=\left(\frac{\alpha_{2}}{\alpha_{1}}, 1\right)$ and $v_{2}=(-1,1)$. A saddle point the attractor of which is $v_{2}$

- $\tilde{G}=\left(\frac{\alpha_{2} \alpha_{3}}{\alpha_{1} \alpha_{2}+\alpha_{2} \alpha_{3}+\alpha_{1} \alpha_{3}}, \frac{\alpha_{1} \alpha_{3}}{\alpha_{1} \alpha_{2}+\alpha_{2} \alpha_{3}+\alpha_{1} \alpha_{3}}\right)$. The eigenvalues are equal and negative, $\lambda_{1}=$ $\lambda_{2}=\frac{-\beta \alpha_{1} \alpha_{2} \alpha_{3}}{\alpha_{1} \alpha_{2}+\alpha_{2} \alpha_{3}+\alpha_{1} \alpha_{3}}<0$ and the eigenvectors are $v_{1}=(1,0), v_{2}=(0,1)$. A stable point in the interior of the $n-1$ simplex to which all the trajectories arrive from all directions.

The graphical representation of the phase diagram is similar to that seen for the other price-setting routine. This result reinforces the idea that the model is robust against changes in the specific pricing routines, number of firms, and initial conditions and parameters. Therefore, we have one stable point, $\tilde{G}$, to which all the trajectories of the system that are not at the boundary of the $n-1$ dimensional simplex tend to go, that is, that $1 \neq s_{i}(0) \neq 0$ for $i=1,2$ and $s_{1}(0)+s_{2}(0) \neq 1$. Note that, for the case $s_{1}(0)+s_{2}(0)=$ 1, we obtain the same results as for the duopoly case. We will now finish this section by looking at the concentration levels and offering certain welfare remarks that our model suggests.

\subsubsection{Herfindahl index and certain remarks on welfare}

The Herfindahl index in the interior stationary state of our oligopoly case is given by $H=s_{1}^{* 2}+s_{2}^{* 2}+$ $s_{3}^{* 2}$ where $s_{1}^{* 2}, s_{2}^{* 2}$ and $s_{3}^{* 2}$ are the market shares in the interior equilibrium. Substituting the different limit-state shares for the alternative pricing routines, we find:

$$
\begin{aligned}
& \widehat{H}=\hat{s}_{1}^{* 2}+\hat{s}_{2}^{* 2}+\hat{s}_{3}^{* 2}=\left(\frac{\eta_{1}}{\eta_{1}+\eta_{2}+\eta_{3}}\right)^{2}+\left(\frac{\eta_{2}}{\eta_{1}+\eta_{2}+\eta_{3}}\right)^{2}+\left(\frac{\eta_{3}}{\eta_{1}+\eta_{2}+\eta_{3}}\right)^{2}= \\
& =\frac{\eta_{1}^{2}+\eta_{2}^{2}+\eta_{3}^{2}}{\left(\eta_{1}+\eta_{2}+\eta_{3}\right)^{2}} ; \\
& \widetilde{H}=\tilde{s}_{1}^{* 2}+\tilde{s}_{2}^{* 2}+\tilde{s}_{3}^{* 2}=\left(\frac{\alpha_{2} \alpha_{3}}{\alpha_{1} \alpha_{2}+\alpha_{2} \alpha_{3}+\alpha_{1} \alpha_{3}}\right)^{2}+\left(\frac{\alpha_{1} \alpha_{3}}{\alpha_{1} \alpha_{2}+\alpha_{2} \alpha_{3}+\alpha_{1} \alpha_{3}}\right)^{2}+ \\
& +\left(\frac{\alpha_{1} \alpha_{2}}{\alpha_{1} \alpha_{2}+\alpha_{2} \alpha_{3}+\alpha_{1} \alpha_{3}}\right)^{2}=\frac{\alpha_{2}^{2} \alpha_{3}^{2}+\alpha_{1}^{2} \alpha_{3}^{2}+\alpha_{1}^{2} \alpha_{2}^{2}}{\left(\alpha_{1} \alpha_{2}+\alpha_{2} \alpha_{3}+\alpha_{1} \alpha_{3}\right)^{2}} .
\end{aligned}
$$


The Herfindahl index in this case will be at a minimum when it is equal to $1 / 3$. This minimum will be reached if and only if the firms have the same price-fixing parameters, that is, $\eta_{1}=\eta_{2}=\eta_{3}$ for the first price-fixing routine (4), and $\alpha_{1}=\alpha_{2}=\alpha_{3}$ for the second one (5). This is a highly improbable situation. Notice also that the monopoly situations in which $H=1$ can generally be ruled out as unstable points of the systems. Therefore, systems (19) and (20) tend to stabilize around a moderate level of industrial concentration. Likewise, and given that prices for the three firms must be identical at the interior resting point, their profits will depend on market shares. Again, if we assume that firms will differ in routines but all of them tend to seek profits (they are not maximizers but profit seekers), if the oligopoly stabilizes at an interior resting point, with relatively evenly-distributed shares (Figure 2), then we can easily obtain settings in which firms compete with a non-extreme level of industrial concentration, the market provides firms with extra-profits, and consumers all get the same payoff at the interior resting point $\hat{u}^{*}=-\hat{p}^{*}$ or $\tilde{u}^{*}=-\tilde{p}^{*}$. Although the final payoff will depend on the limit-prices, we can easily put forward configurations in which the limit-price vector and consumer payoffs are not extremely high. In Figure 2, for plausible parametric values, we obtain a highly evenly-distributed market (low $H$ ) with moderate prices in the interior of the $n-1$ dimensional simplex, positive firm extra profits, and an acceptable level of consumer welfare $\hat{u}^{*}=-\hat{p}^{*}$.

The results obtained in this Section 3 are the basis from which we can fruitfully compare the evolutionary approach to the standard neoclassical static representations (Section 4). Furthermore, the basic setting in Section 3 serves as a platform from which we can move (in Section 5) towards generalized versions of the model.

\section{Our evolutionary model versus the 'Bertrand' neoclassical game}

In this section, we adapt the basic evolutionary model seen in the previous sections to compare its results with a fully-rational (and perfect knowledge) neoclassical "Bertrand-game”. To this regard, we consider two firms that compete in prices, with unitary costs equal to one, and with no significant product differences (perfect or almost perfect substitutes). Firms have pricing capacity, the size of the sector is constant, and, in normalized terms, we have a firm-specific normalized profit function, which is:

$$
\pi_{i}=\left(p_{i}-1\right) s_{i}, \quad i=1,2
$$

Notice that this function (23) is analogous to equation (1). In the Bertrand setting, firms decide their prices, but this time they do not decide upon them by using pricing-routines, but by choosing rationally among strategies, with perfect information, perfect knowledge of the game, and full knowledge of the complete 
payoff matrix. Consumers also have perfect information, and are capable of placing the firms' offers (prices on the products) into a complete and consistent order. Then, they buy the product (from the firm) with the lowest price. We will adapt parametrically the Bertrand setting to obtain results that can be compared directly with those obtained for the alternative pricing routines (4) and (5) in the evolutionary setting. We shall focus first on the "one-shot” version of the Bertrand game.

Thus, we present the payoff matrices of our Bertrand duopoly in Tables 1 and 2, respectively. The duopolist 1 is the row player, and duopolist 2 the column player. We can see that it is a prisoners' dilemma type of frame. The strategies available to the duopolists are simply to cooperate (if both cooperate they collude and agree on fixing a high price as high as possible, depending on the context), or not cooperate (one of them may defect, and, in the extreme case, both can defect). If both firms defect, or even if just one of them defects thus breaking the cooperative agreement, then the duopolists unchain a price war to capture consumers. As we will see, this situation is an attractive (stable) state in the one-shot game. Formally, let's see what payoff each alternative strategy offers and then we can set the payoff matrices:

- Both duopolists collude in the market in such a way that they set the same price, thus obtaining the same share and identical positive profit, with market shares $s_{1}=s_{2}=0.5$, and a profit that is higher, the higher the prices on which firms base the cooperative agreement. Since firms are fully rational, they may try to fix significantly high prices in the collusive state (with an erosion of consumer welfare). We can consider that they agree to set the maximum possible price in accordance with the routines (4), (5); i.e. $\left(\hat{p}_{i}=\frac{\eta_{i}}{\eta_{i}-1}\right.$ or $\left.\tilde{p}_{i}=1+\alpha_{i}\right)$. We assume that, because of firm homogeneity, both firms have the same price-fixing parameter that now, in a typical industrial organization setting, we can consider to be a strategic choice $\left(\eta \equiv \eta_{1}=\eta_{2}>1\right.$ or $\alpha \equiv$ $\alpha_{1}=\alpha_{2}>0$ ). In the case that we want to compare results with the evolutionary setting from routine (4), we can assume that both firms fix prices as $\hat{p}_{i}$. Then, the prices to be set will be $\hat{p}_{1}=$ $\hat{p}_{2}=\frac{\eta}{\eta-1}$. If they use $\tilde{p}_{i}, \tilde{p}_{1}=\tilde{p}_{2}=1+\alpha$, they already represent the maximum price that can be set from equations (4) or (5). In the collusive state, both firms agree to keep an identical (high) price, and thus they split the market share, $s_{1}=s_{2}=0.5$ (Tables 1 and 2).

This is basically a situation with profit-sharing, which leads to normalized profits $\hat{\pi}_{1}=\hat{\pi}_{2}=$ $\left(\frac{\eta}{\eta-1}-1\right) 0.5=\frac{0.5}{\eta-1}$ or $\tilde{\pi}_{1}=\tilde{\pi}_{2}=(1+\alpha-1) 0.5=0.5 \alpha$, depending on the pricing routine. Thus we see that the first cell of the payoff matrix (in Tables 1 or 2), where both firms agree and 
cooperate, is $\left(\frac{0.5}{\eta-1}, \frac{0.5}{\eta-1}\right)$ if prices are to be comparable with $\hat{p}_{i}$, and $(0.5 \alpha, 0.5 \alpha)$ if they are set so that we can compare them with $\tilde{p}_{i}$.

- If the firms betray each other and enter into a price war, they will end up with the same prices at the minimum level to capture consumers. Since both firms' unit cost is one, we have $p_{1}=p_{2}=1$ as the minimum price. Firms make identical profits (null extra profits) $\pi_{1}=\pi_{2}=$ 0 , such that the fourth cell (bottom right) of the payoff matrices (Tables 1 and 2 ) will be $(0,0)$.

- If one of the duopolists unilaterally decides to move away from the agreed-upon colluded solution-assuming that the other firm continues to play this solution — then, as demand reacts to price in our model, this firm only has to set a price slightly lower than the agreed price to be able to corner all the market and make extra-profits. In the case to be compared with routine $\hat{p}_{i}$, player $i$, who decides to betray the other one, slightly increases the parameter $\eta_{i}$, so that, we have $\eta_{i}>$ $\eta$, and then $\hat{p}_{i}=\frac{\eta_{i}}{\eta_{i}-1}<\frac{\eta}{\eta-1}=\hat{p}_{j}$, thus capturing all the market share in the "one-shot" game and obtaining a profit $\hat{\pi}_{i}=\frac{1}{\eta^{+}-1}$. The other duopolist must exit the market in the one-shot version (represented in Table 1 by $0^{-}$). As the game is symmetric, the profits for both duopolists in the top right and lower left cells are those in Table 1 . In the comparable frame with routine $\tilde{p}_{i}$, player $i$, who decides to betray the other one, will slightly decrease parameter $\alpha_{i}$, with respect to the agreed one (that is, $\alpha_{i}<\alpha$ ), $\tilde{p}_{i}=1+\alpha_{i}<1+\alpha=\tilde{p}_{j}$. In this manner, the firm will reach all the market share and will obtain a profit $\tilde{\pi}_{i}=\alpha^{-}$. The other duopolist must exit the market. As the game is symmetric, the profits for both duopolists appear in Table 2.

\begin{tabular}{lccc}
\hline & \multicolumn{2}{c}{ Player 2} \\
& Cooperate & Defect \\
Player 1 & Cooperate & $\left(\frac{0.5}{\eta-1}, \frac{0.5}{\eta-1}\right)$ & $\left(0^{-}, \frac{1}{\eta^{+}-1}\right)$ \\
& Defect & $\left(\frac{1}{\eta^{+}-1}, 0^{-}\right)$ & $(0,0)$ \\
\hline
\end{tabular}

Table 1. Payoff matrix for routine $\hat{p}_{i}\left(\eta^{+}>\eta>1\right)$ 
Note in Table 1 that the non-cooperative (lowest prices by betrayal) "price war" strategy is strongly dominant for both players if we suppose $\frac{1}{\eta^{+}-1}>\frac{0.5}{\eta-1}$. Actually, this supposition is true if and only if $2 \eta-$ $1>\eta^{+}$. Writing $\eta^{+}=\eta+\varepsilon$ with $\varepsilon>0$ our supposition is fulfilled if $\eta-1>\varepsilon$. As $\eta>1$, then $\varepsilon$ will always exist and we can consider that $\frac{1}{\eta^{+-1}}>\frac{0.5}{\eta-1}$ for $\eta^{+}>\eta$. Hence we have a Nash-stable equilibrium of the game, which is that of price war. Even though more is gained by both firms in the collusive state, in the Nash "price war” the Bertrand equilibrium is fully-rational for both firms starting out a price war, which will end up in the Bertrand zero extra-profits state. The other situations of the game (it can be shown that there are no Nash equilibria for mixed strategies) are those associated with one player cooperating while the other does not. However, these states are unstable, and they will lead dynamically to a situation where the firm that has not betrayed the other one lowers its price, which would drag the case to the Bertrand equilibrium $(0,0)$. The (defect, defect) equilibrium is stable.

\begin{tabular}{cccc}
\hline & & \multicolumn{2}{c}{ Player 2 } \\
& & Cooperate & Defect \\
Player 1 & Cooperate & $(0.5 \alpha, 0.5 \alpha)$ & $\left(0^{-}, \alpha^{-}\right)$ \\
& Defect & $\left(\alpha^{-}, 0^{-}\right)$ & $(0,0)$ \\
\hline
\end{tabular}

Table 2. Payoff matrix for routine $\tilde{p}_{i}\left(0<\alpha^{-}<\alpha\right)$

For the matrix in Table 2, once again we see the "defect and the rival reacts by lowering price because of betrayal” effect. Defecting from collusion is strongly dominant for both players if we suppose that $\alpha^{-}>$ $0.5 \alpha$. Actually, writing $\alpha^{-}=\alpha-\varepsilon$ with $\varepsilon>0$, the supposition is reduced to $0.5 \alpha>\varepsilon$, and as $\alpha>0$, then $\varepsilon$ will always exist. Thus we see that the (defect, defect) Nash-state is stable and attractive.

To conclude this comparative exercise, we are going to set out from Table 1 and consider what would be the effects on the game if we considered the repeated version (from time " 0 " to infinity). The situation for Table 2 is similar; therefore, we will just carry out the analysis for Table 1. Let us consider for the repeated version that the two fully-rational, fully-informed firms have a common discount rate $r$. Setting out from Table 1, we start out from the collusive situation and obtain the result that the incentives to defect in the initial period exist. If any one of the two firms performs unilateral defection from collusion, it gains an extra profit from betrayal-which we will call the temptation to defect-and (in Table 1) it is equal to 
"what the defector can capture as extra-gain if it breaks cooperation unilaterally whereas the other firm still cooperates". In Table 1, we can see that temptation is $\frac{1}{\eta^{+}-1}-\frac{0.5}{\eta-1}>0$. This temptation is positive, since, as we have seen above, we consider $\eta^{+}>\eta$.

Nonetheless, this gain (the temptation) will only happen in the first period, because defection will generate the reaction of the rival, and the game will quickly (and forever) end up in the Nash-stable state, (defect, defect): $(0,0)$. Therefore, a punishment appears in the repeated game (we assume the trigger strategy), which the potential defector must compare with the temptation so as to decide whether defecting pays or not. If temptation is higher than punishment, we will have defection and a price war in the repeated game. Punishment means moving from the cooperative solution to the Nash-stable state forever. The potential defector (Table 1) may then calculate the punishment (defecting) as being: $\sum_{i=1}^{\infty} \frac{1}{(1+r)^{i}} \frac{0.5}{\eta-1}=\frac{0.5}{r(\eta-1)}>0$. From the previous results, we can obtain the result that temptation is higher than punishment, thus leading to a price war, when we have: $\frac{1}{\eta^{+}-1}>\left(1+\frac{1}{r}\right) \frac{0.5}{\eta-1}$. We already know that $\frac{1}{\eta^{+}-1} / \frac{0.5}{\eta-1}>1$ but it is not clear whether we will have:

$$
\frac{1}{\eta^{+-1}} / \frac{0.5}{\eta-1}>1+\frac{1}{r}
$$

This is the condition for breaking cooperation and entering in a price war. The higher the discount rate " $r>0$ ", the more probable it is that the sector ends up in a price war. On the other hand, a value of " $r$ ", which is sufficiently low, rules out the price war à la Bertrand, but then it ensures—in the repeated gamea permanent situation of collusion with prices that are too high and market sharing, a situation that is clearly detrimental for consumer welfare.

It is interesting to remark that, if we compare the evolutionary results with the Bertrand game, we see how in the evolutionary model, from both the point of view of firms (which are boundedly rational) and also from the point of view of consumers (who gradually learn better price options), we obtain dynamics converging to a stable interior equilibrium where firms end up obtaining extra-profits and compete with moderate prices, and consumers do not see their welfare eroded by excessively high prices (see the generalization in Section 5). By contrast, within the Bertrand game (with full rationality and perfect knowledge), firms either do not obtain extra-profits (price war) or, if they maintain a collusive agreement with higher prices, then they significantly erode consumer welfare. Now, in Section 5, we are going to 
extend the evolutionary model for the general case of $n$ firms, and to the case of heterogeneous firm costs and technological advance.

\section{Extensions of the evolutionary model}

\subsection{The general case of " $n$ " firms.}

We first extend the analysis of the evolutionary model composed of equation (9) and the pricing routines (4) and (5) to the case of any number of firms $(i=1,2, \ldots, n)$. It is crucial to note that, if we had not analyzed the model "step by step” and in a comparative manner (as we have done in Sections 3 and 4), it would have been impossible to reach the general results we hereby present. The low-dimensional comparative analyses provided us with a lot of intuition and preparatory information that we will need now. Thus, we will look at the " $n$ ” firms general case. We carry out the exhaustive formal analysis for the pricing routine (5) (but the results are qualitatively identical for routine (4) since, as we know, routine (5) can be reached through a Taylor expansion of (4) and both are almost identical for positive real values between " 0 ” and " 1 ”, which is the case of market shares). We have chosen routine (5) here because it requires fewer calculations.

Then, considering equation (9) and the routine (5) we can re-write the model for any $n$ as follows:

$$
\begin{aligned}
\dot{s}_{l}=\beta s_{i}(t)\left[\sum_{j=1}^{n} s_{j}(t) p_{j}(t)-p_{i}(t)\right] \\
p_{i}(t)=1+\alpha_{i} s_{i}(t), \quad(i=1,2, \ldots, n) .
\end{aligned}
$$

The dynamics of (24) develop on the unit-simplex $\Delta^{n}$ and both the boundary and the interior of $\Delta^{n}$ are invariant sets (Sandholm 2010). We are going to focus on the interior of the simplex, although, of course, it is clear that we still have rest points on the faces, edges, and vertices of the simplex. However, since the interior is invariant, and we always run the analysis from initial conditions within the interior of the simplex (let us denote this interior by $D=\operatorname{Int}\left(\Delta^{n}\right)$ ), we are interested in the existence and stability of interior resting points. Moreover, the results obtained for the interior of $\Delta^{n}$ will be the ones we must consider to see whether our results in Section 3 can be generalized for the $n$-case. The proposition 1 below synthesizes the key generalizations for the fully general $n$-case:

\section{Proposition 1}

The general replicator system (24) with pricing routine (5) has a unique equilibrium point 
$s^{*} \in D=\operatorname{Int}\left(\Delta^{n}\right)$. This point $s^{*}$ is globally asymptotically stable within $D$. Therefore, by proving this proposition, we are verifying the generality of our results in previous sections for the n-case.

\section{$\underline{\text { Proof }}$}

1) First, inspired by the analysis in Section 3, we find that there is always at least an interior resting point $s^{*} \in D=\operatorname{Int}\left(\Delta^{n}\right)$ for (24), which, in clear concordance with the previous sections, is given by:

$$
s^{*}=\left(s_{1}^{*}, s_{2}^{*}, \ldots, s_{n}^{*}\right)=\left(\frac{\alpha_{2} \alpha_{3} \ldots \alpha_{n}}{\sum_{i} \frac{\left(\alpha_{1} \alpha_{2} \ldots \alpha_{n}\right)}{\alpha_{i}}}, \frac{\alpha_{1} \alpha_{3} \ldots \alpha_{n}}{\sum_{i} \frac{\left(\alpha_{1} \alpha_{2} \ldots \alpha_{n}\right)}{\alpha_{i}}}, \ldots, \frac{\alpha_{1} \alpha_{2} \ldots \alpha_{n-1}}{\sum_{i} \frac{\left(\alpha_{1} \alpha_{2} \ldots \alpha_{n}\right)}{\alpha_{i}}}\right)
$$

It is straightforward to see that, if we substitute this expression in (24), we obtain that in $s^{*}, \dot{s}_{l}=0, \forall i$ is always verified.

2) Second, to guarantee that our results in Section 3 are robust and valid for any $n$, we have to check also the stability of $s^{*}$. In fact, since we are going to verify that $s^{*}$ is globally asymptotically stable within $D$, we also see that $s^{*}$ is the unique equilibrium within $D$. We proceed by using the direct Lyapunov method of proof (Weibull 1995). Thus, we have defined $D=\operatorname{Int}\left(\Delta^{n}\right)$ which is an open invariant set, in fact it is an invariant neighborhood of the interior equilibrium point $s^{*}$. By using the Lyapunov method, we can assure that $s^{*}$ is unique and globally asymptotically stable within $D$ if we find a continuous and differentiable function $V: D \rightarrow R$ that verifies the following properties in $D$ :

i) $\quad V(s)=0 \Leftrightarrow s=s^{*}$, and $V(s)>0 \quad \forall s \neq s^{*}$ in $D$.

ii) $\forall s \neq s^{*}$ in $D$, and we also see that $\frac{d V(s)}{d t}<0$.

For our general system (24) and the pricing routine (5) $p_{i}(t)=1+\alpha_{i} s_{i}$, we can prove that $V(s)=$ $-\sum_{i=1}^{n} \ln \left(\frac{p_{i}(t)}{\bar{p}(t)}\right)=-\sum_{i=1}^{n} \ln \left(\frac{1+\alpha_{i} s_{i}(t)}{\sum_{i=1}^{n} s_{i}(t)\left(1+\alpha_{i} s_{i}(t)\right)}\right)$ is a strict Lyapunov function in $D$, verifying conditions i) and ii). Let us verify this:

*Regarding $i)$, notice that in $s^{*}$ all prices are equal and identical to the average price $\bar{p}(t)$. As a 
consequence, $V\left(s^{*}\right)=-\sum_{i=1}^{n} \ln (1)=0$.

On the other hand, for any $s \neq s^{*}$ within $D, V(s)=-\sum_{i=1}^{n} \ln \left(\frac{p_{i}(t)}{\bar{p}(t)}\right)>0 \Leftrightarrow \sum_{i=1}^{n} \ln \left(\frac{p_{i}(t)}{\bar{p}(t)}\right)<0 \Leftrightarrow$ $\ln \left[\prod_{i=1}^{n}\left(\frac{p_{i}(t)}{\bar{p}(t)}\right)\right]<0 \Leftrightarrow e^{\ln \left[\prod_{i=1}^{n}\left(\frac{p_{i}(t)}{\bar{p}(t)}\right)\right]}<e^{0}(=1)$. It is clear that this is verified if and only if:

$$
\prod_{i=1}^{n}\left(\frac{p_{i}(t)}{\bar{p}(t)}\right)<1
$$

This expression is equivalent to proving that $\left(p_{1}(t) \cdot p_{2}(t) \ldots . p_{n}(t)\right)<\bar{p}(t)^{n}$. Then, this expression is true if $\bar{p}(t)>\sqrt[n]{p_{1}(t) \cdot p_{2}(t) \ldots p_{n}(t)}$. We remember that $\bar{p}(t)$ is the weighted average of the prices in the sector, while $\sqrt[n]{p_{1}(t) \cdot p_{2}(t) \ldots . p_{n}(t)}$ is the geometric mean of those prices. With all prices being positive, and considering a non-excessive degree of price dispersion (since we are modeling a sector in which close varieties of the same good are produced and supplied), then we can affirm that $\bar{p}(t)$ is a sufficiently good approximation of the arithmetic mean of the prices. It is well known that, in these conditions, the arithmetic mean is always significantly higher than the geometric mean, and therefore, in our conditions, $\bar{p}(t)>\sqrt[n]{p_{1}(t) \cdot p_{2}(t) \ldots . p_{n}(t)}$ and condition i) (for $V(s)$ to be a strict Lyapunov function) is verified. We can affirm that $V(s)=0 \Leftrightarrow s=s^{*}$ and $V(s)>0 \quad \forall s \neq s^{*}$ in $D$.

*Regarding $\mathrm{ii}$ ), we need to verify first, as a previous step, that for the pricing routine (5) and system (24) it is $\frac{d \bar{p}}{d t}=\frac{d\left(\sum_{i=1}^{n} s_{i}(t) p_{i}(t)\right)}{d t}<0$.

Notice that the average price with this routine is $\bar{p}=1+\sum s_{i}^{2} \alpha_{i}$.

Considering (5) and (24) we take derivatives in $\bar{p}$ :

$$
\frac{d \bar{p}}{d t}=-2 \beta \sum_{i=1}^{n} s_{i}\left(\alpha_{i} s_{i}\right)\left(p_{i}-\bar{p}\right)=-2 \beta \operatorname{Cov}(\alpha s, p)<0
$$

It is clear that this is true because, since $p_{i}(t)=1+\alpha_{i} s_{i}$, the covariance between the prices $\left(p_{i}\right)$ and the margins $\left(\alpha_{i} s_{i}\right)$ is always positive. Therefore,

$$
\frac{d \bar{p}}{d t}<0
$$

Now, by taking derivatives in $V(s)$ we obtain:

$$
\frac{d V(s)}{d t}=-\left[\sum_{i=1}^{n} \frac{\left(d p_{i} / d t\right)}{p_{i}}-n \frac{(d \bar{p} / d t)}{\bar{p}}\right]
$$

We have to prove that this expression is negative in the domain. We know from the previous step (notice 
$\left.\frac{d \bar{p}}{d t}<0\right)$ that $-n \frac{(d \bar{p} / d t)}{\bar{p}}>0$, but we have to study the remaining summand, that is, what can we state regarding the sign of $\sum_{i=1}^{n} \frac{\left(d p_{i} / d t\right)}{p_{i}}$ ? From the pricing routine (5) and system (24) it is clear that:

$$
\sum_{i=1}^{n} \frac{\left(d p_{i} / d t\right)}{p_{i}}=-\beta \sum_{i=1}^{n} s_{i}\left(\frac{\alpha_{i}}{p_{i}}\right)\left(p_{i}-\bar{p}\right)=-\beta \operatorname{Cov}\left(\frac{\alpha}{p}, p\right)>0
$$

We can see that the expression is positive since we have a negative covariance between the prices $(p)$ and something divided by the prices. Then, having found that in our conditions that both $\sum_{i=1}^{n} \frac{\left(d p_{i} / d t\right)}{p_{i}}>0$ and $-n \frac{(d \bar{p} / d t)}{\bar{p}}>0$ we can affirm that:

$$
\frac{d V(s)}{d t}<0
$$

Therefore, we have verified that a strict Lyapunov function exists with the expression: $V(s)=-\sum_{i=1}^{n} \ln \left(\frac{p_{i}(t)}{\bar{p}(t)}\right)=-\sum_{i=1}^{n} \ln \left(\frac{1+\alpha_{i} s_{i}(t)}{\sum_{i=1}^{n} s_{i}(t)\left(1+\alpha_{i} s_{i}(t)\right)}\right)$ for (24) with the routine (5), which assures that the point $s^{*}$ is a unique and globally asymptotically stable resting point within $D=\operatorname{Int}\left(\Delta^{n}\right)$.

Proposition 1 generalizes the results in Section 3 for the " $n$ " case. We present now an illustrative simulation of system (24) and routine (5) to help the reader in understanding that all the results in Section 3 are generalized through proposition 1. Without any loss of generality, we consider the case of $n=5$ (as an illustration of high $n$ ), with parametric values $\alpha_{1}=0.52, \alpha_{2}=0.5, \alpha_{3}=0.6, \alpha_{4}=0.5, \alpha_{5}=0.47, \beta=$ 0.2 , and initial conditions $s_{10}=0.2, s_{20}=0.04, s_{30}=0.45, s_{40}=0.06, s_{50}=0.25$.

The global convergence to $s^{*}=\left(s_{1}^{*}, s_{2}^{*}, \ldots, s_{n}^{*}\right)=\left(\frac{\alpha_{2} \alpha_{3} \ldots \alpha_{n}}{\sum_{i} \frac{\left(\alpha_{1} \alpha_{2} \ldots \alpha_{n}\right)}{\alpha_{i}}}, \frac{\alpha_{1} \alpha_{3} \ldots \alpha_{n}}{\sum_{i} \frac{\left(\alpha_{1} \alpha_{2} \ldots \alpha_{n}\right)}{\alpha_{i}}}, \ldots, \frac{\alpha_{1} \alpha_{2} \ldots \alpha_{n-1}}{\sum_{i} \frac{\left(\alpha_{1} \alpha_{2} \ldots \alpha_{n}\right)}{\alpha_{i}}}\right)$ is shown in Figure 3. Qualitatively identical results can be obtained with the routine (4), exactly as we saw for two and three firms in Section 3. The simulations that we present hereby are simply an illustration, as Proposition 1 is a general result. 


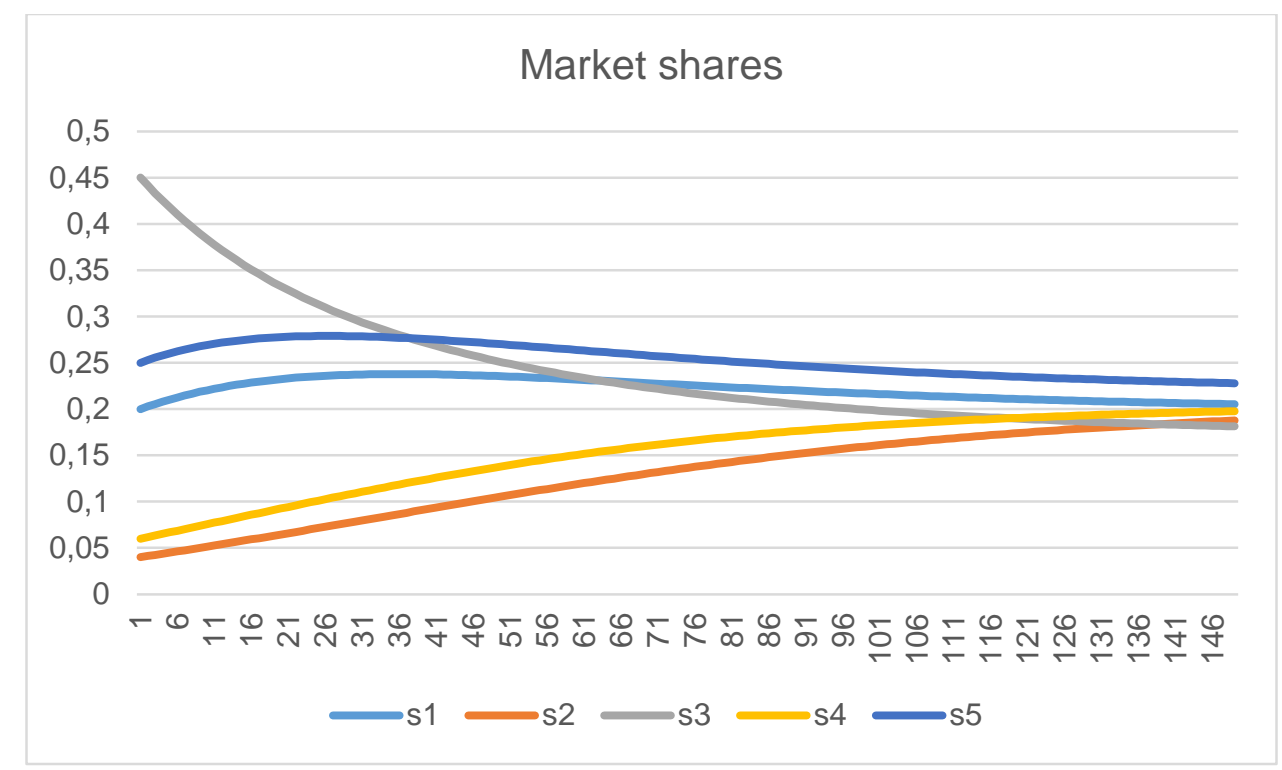

Figure 3. Illustrative simulation of (24) with five firms, convergence to $s^{*}$

In Figures 4, 5, and 6, we show the corresponding trajectories of prices, the average price, and firm profits for the simulation in Figure 3.

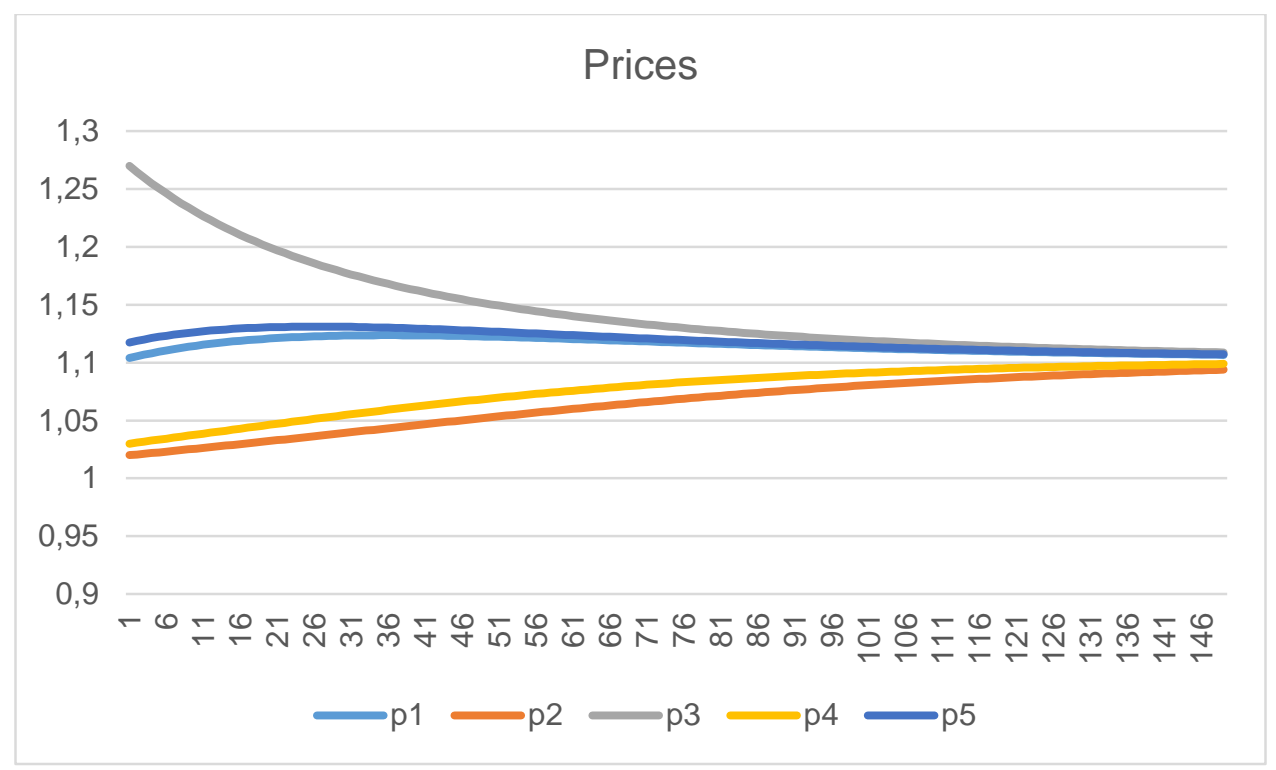

Figure 4. The trajectories of prices during the convergence to $s^{*}$ 


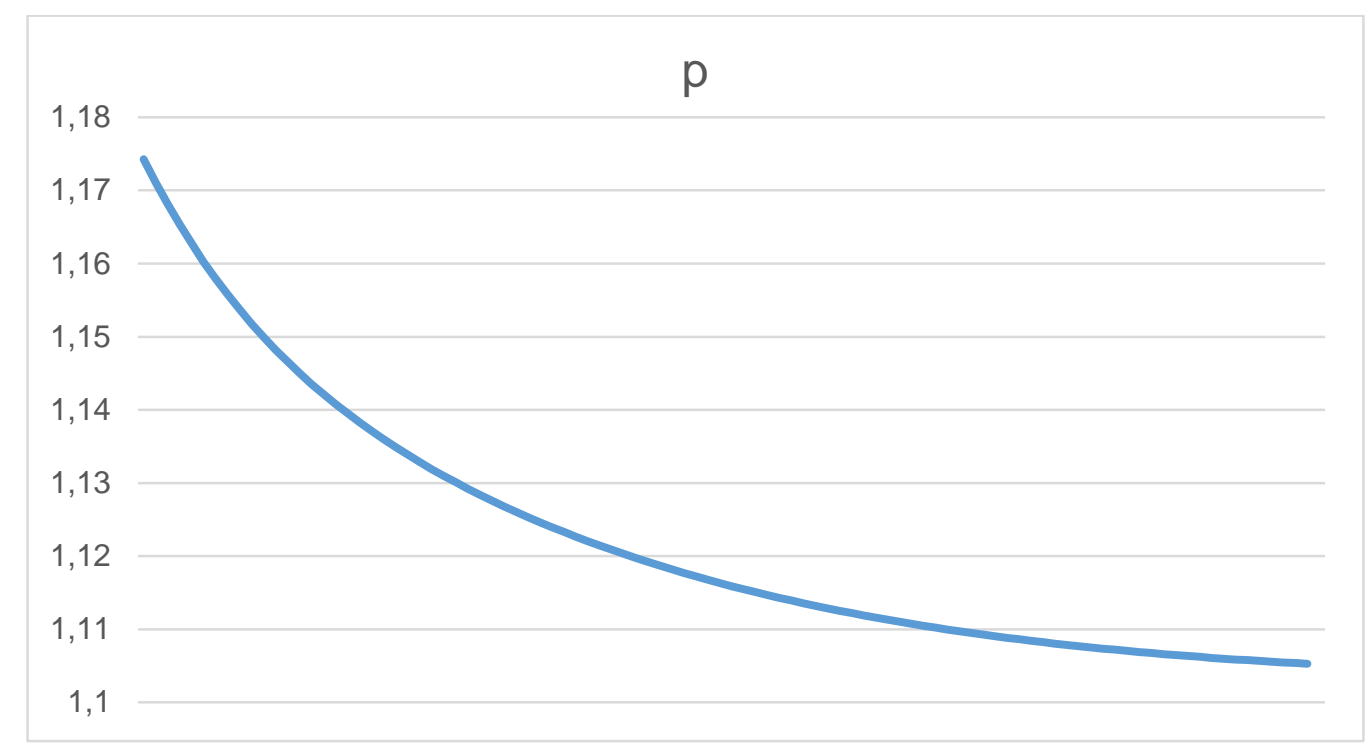

Time: 1 to 100 tending towards 1.1

Figure 5. The decline of the average price during the convergence to $s^{*}$

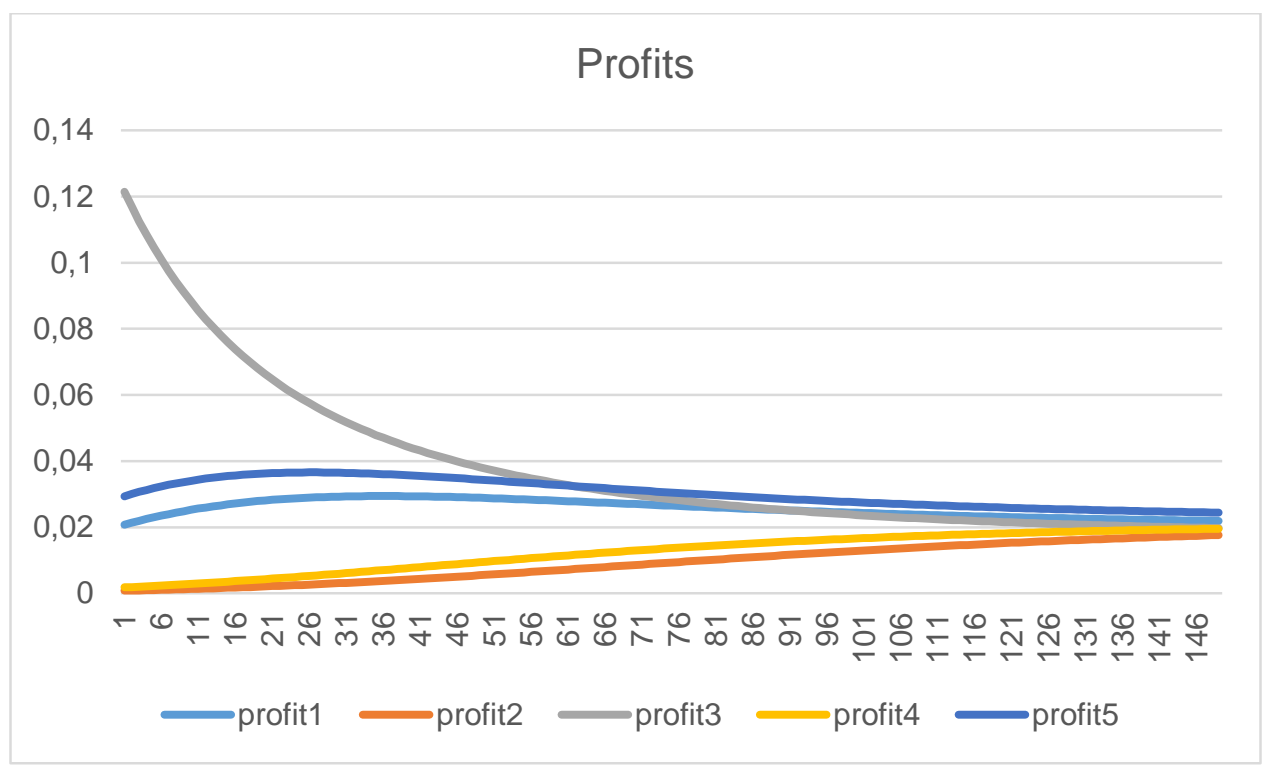

Figure 6. The trajectories of firm profits during the convergence to $s^{*}$

In economic terms, if we wanted to interpret the Proposition 1, we can say that it conveys and generalizes what we have seen in Section 3-regarding market competition in our model as a mechanism for 
generating, discovering, and taking advantage of price differentials. Thus, setting out from any economically relevant initial condition for our general $n$-version (24) (that is to say, starting out from any $s \neq s^{*}$ in $D$ no matter where we use routine (4) or (5)) there is always a potential of welfare improvement through market trading both for consumers and firms. This information is being generated, updated, indicated by prices, imperfectly perceived by consumers, and regenerated step by step as time goes by. The market gradually generates, indicates, and takes advantage of this potential as time passes. The dynamics of supply-demand interactions in our model revealed that this potential tends to be gradually exhausted. The market evolves and tends towards $s^{*}$ (see Figures 3 to 6).

This process resembles the typical Austrian representation of market competition as a discovery process. Moreover, regarding the literature on evolutionary games, and bringing out the comparative analysis performed in Section 4, it is interesting to highlight that the point $s^{*}$ is a Nash-equilibrium stable state in our evolutionary model. Thus, neither consumers nor firms gain anything if either individually or in small masses they decide to move unilaterally away from $s^{*}$. Firms would either erode their extra-profits (see Figure 6) going into a price war if they decided to lower prices, or unilaterally lose customers if they increased prices. In the case of consumers, if a mass of them decided to abandon $s^{*}$ they would generate a price increase in the landing firm (which erodes their welfare as compared with Figure 4), and they would generate a reduction in the price of the original firm. This situation would be reversed quickly, thereby restoring point $s^{*}$. Notice, however, that the limit point $s^{*}$ in our evolutionary model represents a very different type of situation when compared with the Nash-stable state in the Bertrand neoclassical game. As we have seen when comparing Sections 3 and 4, and now in Figures 3 to 6, our evolutionary market makes extra-profits even at the limit; firms tend to co-exist by sharing the market but they do not necessarily have identical shares; and the process tends to $s^{*}$ by decreasing the average price and equalizing firm prices, thus generating an ongoing gain of consumer welfare (with no danger of collusion). This is very different from the zero extra-profits and identical sharing of the Bertrand game, or from the collusion potential result in the repeated Bertrand game.

Of course this first extension of the model opens a whole research line (by incorporating innovation, performance, and institutions in this simple model) along the lines of what we have explored in Almudi et al. (2012, 2013) or Fatas-Villafranca et al. (2009). Still, we believe that the step-by-step analytical process through which we have reflected on the nature of markets as information processors, and the comparisons with the neoclassical Bertrand game are essential. In fact, we can note that the Bertrand result in Section 4 (no extra-profits, identical sharing of the market) might be obtained (as a very special concrete 
case of our evolutionary model) if we ruled out the dynamics and focused just on the static point $s^{*}$ for the very special case in which $n \rightarrow \infty$. As a future research line, we can compare classic results in repeated games with threats (applying Folk theorems) with the solutions from our evolutionary model. But the

explorations of these possibilities exceed the scope of this single paper. Therefore, we leave aside these reflections for future works, and we will finish this paper by considering the evolutionary case with cost heterogeneity and process innovation affecting the dynamics of unit costs.

\subsection{Heterogeneous costs and technological advance}

In this extension, we will work with the pricing routine (2) with unit costs different from "1" (although everything can be extended to the case of routine (1) since both are dynamically equivalent). We can rewrite the original system as follows:

$$
\begin{aligned}
\dot{s}_{l} & =\beta s_{i}(t)\left[\sum_{j=1}^{n} s_{j}(t) p_{j}(t)-p_{i}(t)\right] \\
& p_{i}(t)=\frac{\eta_{i} c}{\eta_{i}-s_{i}(t)}, \quad \eta_{i}>1 \quad(i=1,2, \ldots, n) .
\end{aligned}
$$

The extension we now present considers that, instead of using a common and constant firm unit-cost $c$, we incorporate in the pricing routine (2) a changing firm specific unit cost $c_{i}(t)$.

Then we have the system:

$$
\begin{aligned}
\dot{s}_{l} & =\beta s_{i}(t)\left[\sum_{j=1}^{n} s_{j}(t) p_{j}(t)-p_{i}(t)\right] \\
& p_{i}(t)=\frac{\eta_{i} c_{i}(t)}{\eta_{i}-s_{i}(t)}, \quad \eta_{i}>1 \quad(i=1,2, \ldots, n) .
\end{aligned}
$$

We need to specify a plausible expression for $c_{i}(t)$. Thus, we can assume a firm specific production function (with full use of labor as the main input and elastic labor supply) such as $Q_{i}(t)=q_{i}(t) L_{i}(t)$, in which $q_{i}(t)$ represents labor productivity. Likewise, we can assume that there is raw material used in production the price and technical coefficient of which are common and constant among firms. We can assume that the unit raw material cost is denoted by $\psi>0$, and represents the cost per unit of output of specialized minerals or energy, or other materials bought at a constant and common price in large international markets. This is a characteristic that is plausible for such high tech sectors as microelectronics, pharmaceutical biotechnology, genomics. We may state the total cost of each firm as given by:

$$
T C_{i}(t)=w L_{i}(t)+\psi Q_{i}(t)
$$

If we use the wage as numerairy $w=1$ (see Section 2) and, without loss of generality, we consider the unit 
raw material cost in wage units as also being equal to one, we can obtain each firm's unit cost at any time as follows:

$$
c_{i}(t)=\frac{L_{i}(t)}{Q_{i}(t)}+1=1+\frac{1}{q_{i}(t)}, \quad q_{i}(t) \text { is labor productivity. }
$$

In this manner, we can introduce cost heterogeneity in (26). In fact, we go a step further and we can also include certain dynamics of technological advance. We propose a function for the time-path of each firm's labor productivity as follows:

$$
q_{i}(t)=1+e^{s_{i}(t) t-b_{i}}
$$

Abundant literature supporting this type of exponential productivity growth pattern can be found, from different perspectives and commented on by several different authors, in the overview by Nelson (2018). In (27), $s_{i}(t)$ is the firm's market share, $b_{i}>1$ is a firm specific parameter capturing organizational rigidity when assimilating technological advances (its value may be related with the organizational difficulties in learning and updating processes), and $t \in[0, \infty$ [ is the variable time. The market share incorporates a success-breeds-success (dynamic) increasing returns component in the unit cost. If we introduce equation (27) in the expression for firm unit cost we obtain:

$$
c_{i}(t)=1+\frac{1}{1+e^{s_{i}(t) t-b_{i}}}
$$

This unit cost function with learning and technological advance has important empirically-relevant properties. Thus, $c_{i}(0)=1+\frac{1}{1+e^{-b_{i}}}>1$, and we also see in (28) that the $\lim c_{i}(t)=1$ is a function that is monotonically decreasing with time (with an asymptotic lower bound in " 1 ” for comparability with previous sections), and it shows an inverse S-shaped profile (first strictly concave, then strictly convex) with a time-inflection point at $t=\frac{b_{i}}{s_{i}}$. The endogenous (and evolving) component $s_{i}(t)$ appears as a success-breeds-success mechanism pointing to the fact that those firms that gain share and profitability in our model achieve more resources to try to accelerate learning and technological advance. We can also identify equation (28) with a type of learning curve with labor enhancing technological advance, a successbreeds-success mechanism, and a firm specific rigidity component $b_{i}$. The inverse S-shaped (decreasing) pattern resembles those paths recently found in genomics and bio-pharm industries, and it can also be related to the well-known cost-reduction paths observed in semiconductors, computers or the automobile industries (see Dosi et al. 1988, or, more recently, Nelson 2018).

If we now introduce (28) in (27) we obtain the following Schumpeterian dynamic system: 


$$
\begin{aligned}
\dot{s}_{l} & =\beta s_{i}(t)\left[\sum_{j=1}^{n} s_{j}(t) p_{j}(t)-p_{i}(t)\right] \\
p_{i}(t) & =\frac{\eta_{i}}{\eta_{i}-s_{i}(t)} c_{i}(t), \quad \eta_{i}>1, \quad(i=1,2, \ldots, n), \\
c_{i}(t) & =1+\frac{1}{1+e^{s_{i}(t) t-b_{i}}}
\end{aligned}
$$

It is worth noting that, if we substitute $c_{i}(t)$ in the pricing routine, and we operate, we arrive at a new expression for the price $p_{i}(t)$ that must be introduced into the replicator equation. Thus, we have:

$$
p_{i}(t)=\frac{\eta_{i}}{\eta_{i}-s_{i}(t)}+\frac{\eta_{i}}{\eta_{i}-s_{i}(t)} \frac{1}{1+e^{s_{i}(t) t-b_{i}}}
$$

This expression must be incorporated within the replicator system. Nevertheless, notice that $\hat{p}_{i}(t)=$ $\frac{\eta_{i}}{\eta_{i}-s_{i}(t)}$ is exactly the routine in equation (4), which we used in the analysis for Section 3 . Then, we can re-write the price as:

$$
p_{i}(t)=\hat{p}_{i}(t)+\frac{\hat{p}_{i}(t)}{1+e^{s_{i}(t) t-b_{i}}}
$$

It is very important to realize here that, if we look at a sector with a moderate degree of firm heterogeneity so that the replicator dynamics operate slowly, then as $t \rightarrow \infty$, the second summand $\frac{\hat{p}_{i}(t)}{1+e^{s_{i}(t) t-b_{i}}}$ will have time to almost vanish through technical advance, and the price that will tend to work within the replicator (29) will be exactly the one in Section 3 (equation (4)). In this case, the limit behavior of our extended model with technological change and heterogeneous unit costs is exactly the one we have already analyzed in Sections 3 and 5.1. Of course, the transient dynamics are a little different, but the results will essentially confirm the findings in Sections 3 and 5.1.

As an example of these circumstances in system (29), we show the results of a simulation converging to the globally asymptotically stable interior resting point in Figures 7, 8, and 9. Prices end up converging to a common value, all firms share the market, and they gain positive extra-profits. An example to illustrate this situation that confirms the results in the previous sections would be a setting (for the case of $n=5$ firms) with parametric values $\beta=0.1, \eta_{1}=1.1, \eta_{2}=1.10011, \eta_{3}=1.105, \eta_{4}=1.1, \eta_{5}=1.025, b_{1}=11(i=$ $1, \ldots, 5), s_{10}=0.205, s_{20}=0.2, s_{30}=0.19, s_{40}=0.21, s_{50}=0.195$. We obtain the market evolution that we show in Figures 7, 8, and 9. 


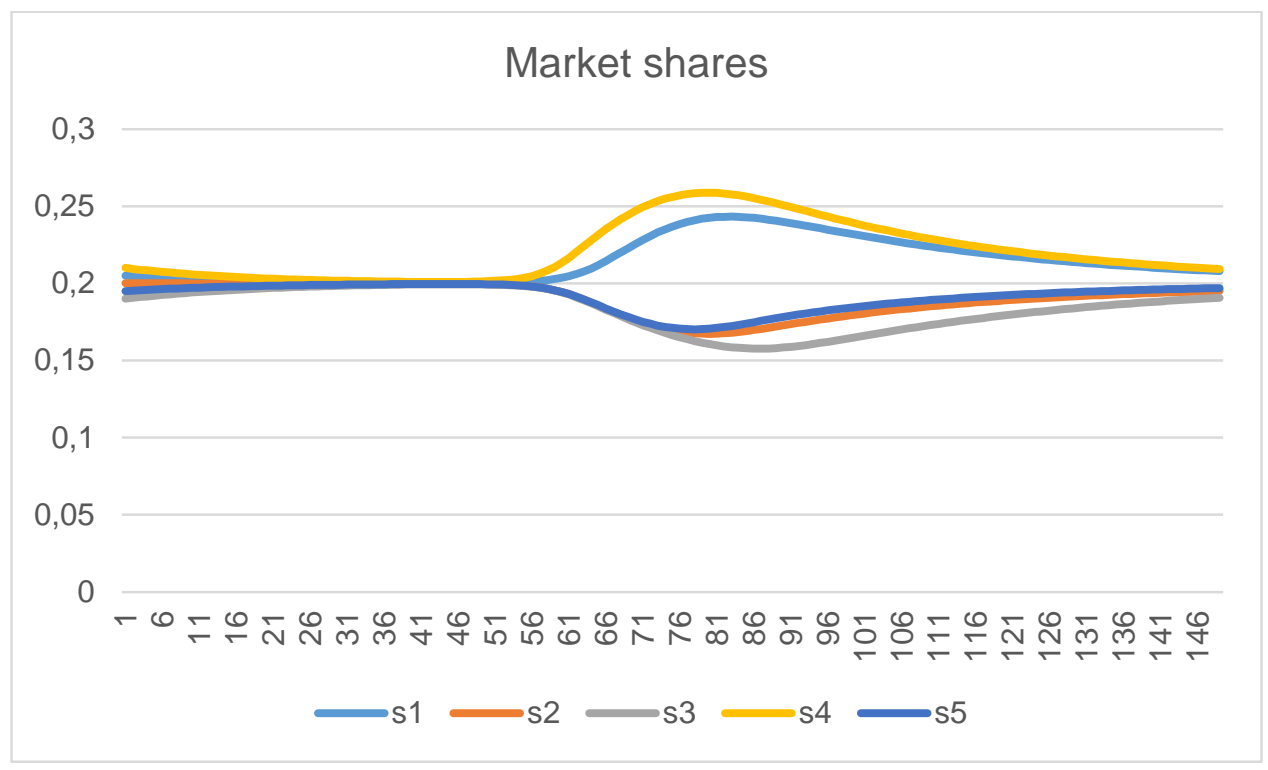

Figure 7. Simulation of (29) with five firms, market shares

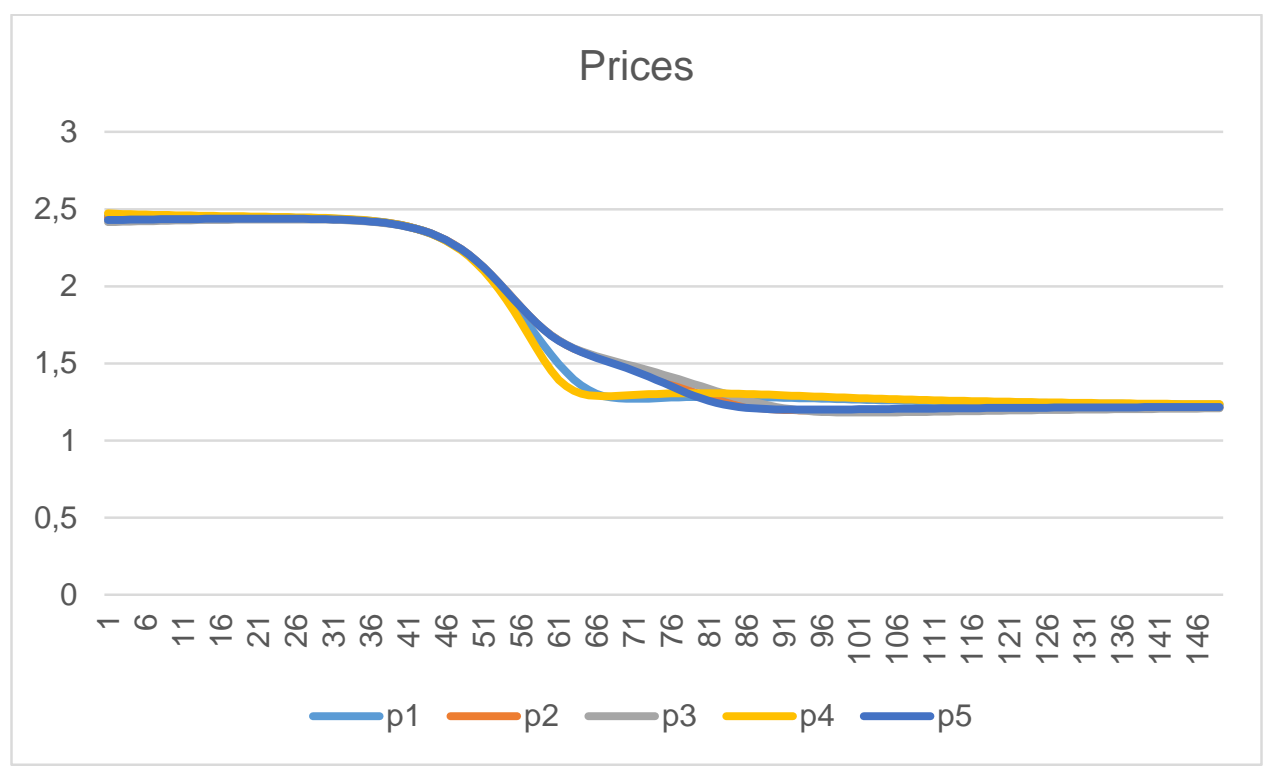

Figure 8. Simulation of (29) with five firms, prices 


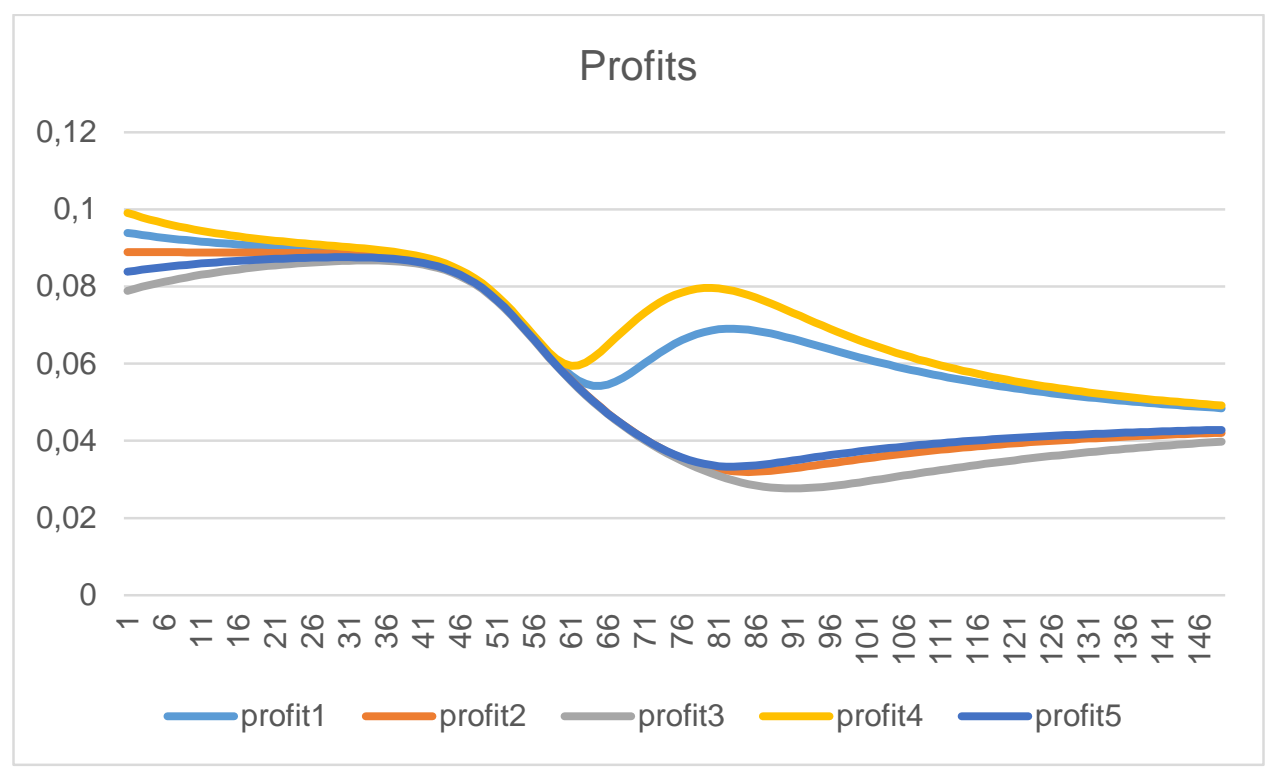

Figure 9. Simulation of (29) with five firms, profits

Apart from other simulation experiments that we could run in the future, in Figures 7, 8, and 9, we essentially obtain a confirmation and generalization of the results obtained in the previous sections. In this simulation, the dynamics have a unique interior asymptotically stable state, in which firms share the market, the level of market concentration tends to be not very high, firms get extra-profits, and prices equalize with a declining average price in the sector so that consumers gain payoff. These are qualitatively identical results to those obtained in Sections 3 and 5.1 departing from the model in Section 2, and they can also be obtained for the other pricing routine.

Nevertheless, in this evolutionary neo-Schumpeterian extension, new possibilities appear. As we increase the degree of heterogeneity and the inter-firm distinct capabilities for technological advance, the transient effects of uneven firm-evolution become very significant, and system (29) does not have time for technical change to erode sufficiently unit costs. In these conditions, we find an enormous variety of dynamic paths (in such a simple model), in which what happens out of equilibrium endogenously separates the system from the interior resting point. In these conditions, even in such a simple evolutionary model, we have market trajectories leading the system either to resting points on the faces of the simplex, or to any of the edges, or even to one of the vertices of the simplex. Despite its simplicity, our evolutionary model is robust to important changes in the specification and also under certain Schumpeterian conditions. However, once we span the array of possibilities in the Schumpeterian specification (and again in spite of the model's simplicity), system (29) can display a wide variety of dynamic patterns. Of course, this finding opens a 
whole line of theoretical advance. In fact, in future works, the ranges delimiting the corresponding outcomes could be analyzed in a closed-form manner. For illustrative purposes, and to close this paper, we now show a case in which we run the Schumpeterian model and the market selects a duopoly. We set out from system (29) with $n=5$ firms, and values $\beta=0.1, \eta_{1}=1.1, \eta_{2}=1.4, \eta_{3}=1.2, \eta_{4}=1.3, \eta_{5}=1.1, s_{\mathrm{i} 0}=$ $0.2(i=1, \ldots, 5), b_{1}=11, b_{2}=14, b_{3}=12, b_{4}=13, b_{5}=14$. We obtain the simulation in Figures 10, 11, and 12 .

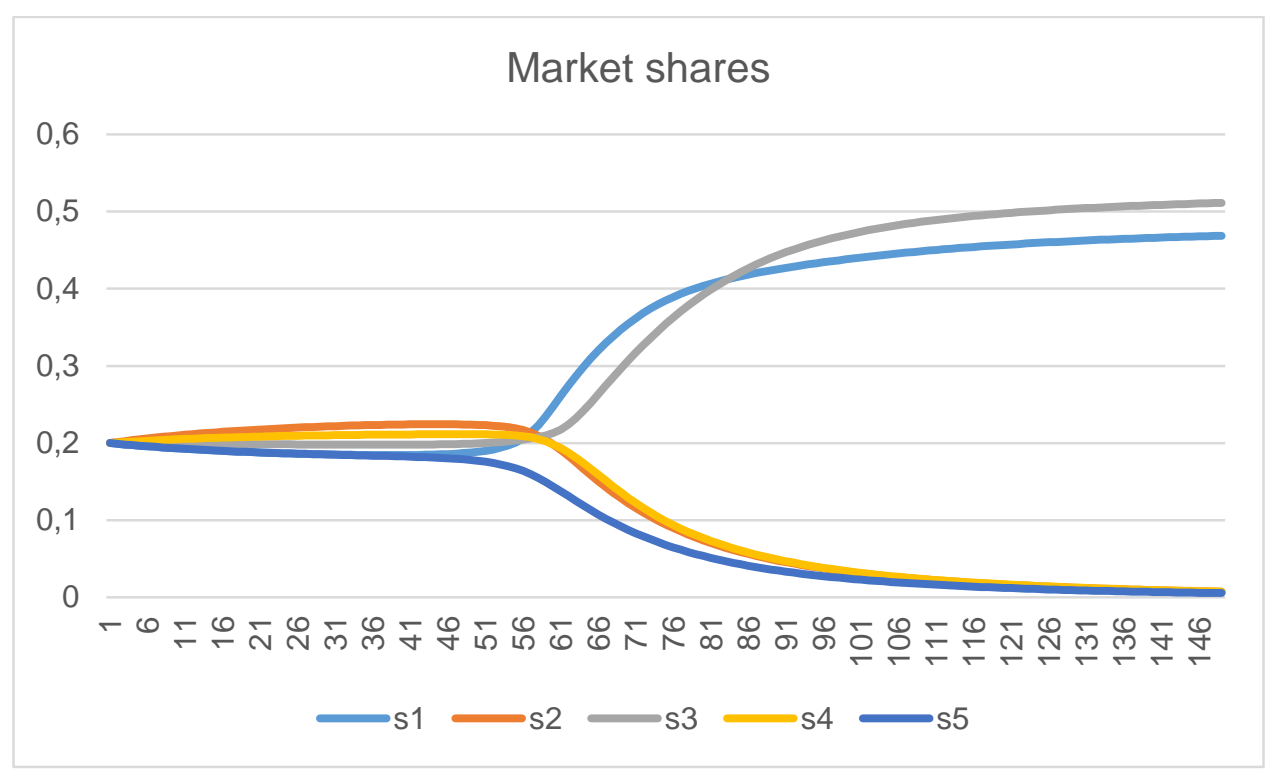

Figure 10. Simulation of (29) with five firms, market shares, non-convergence to interior

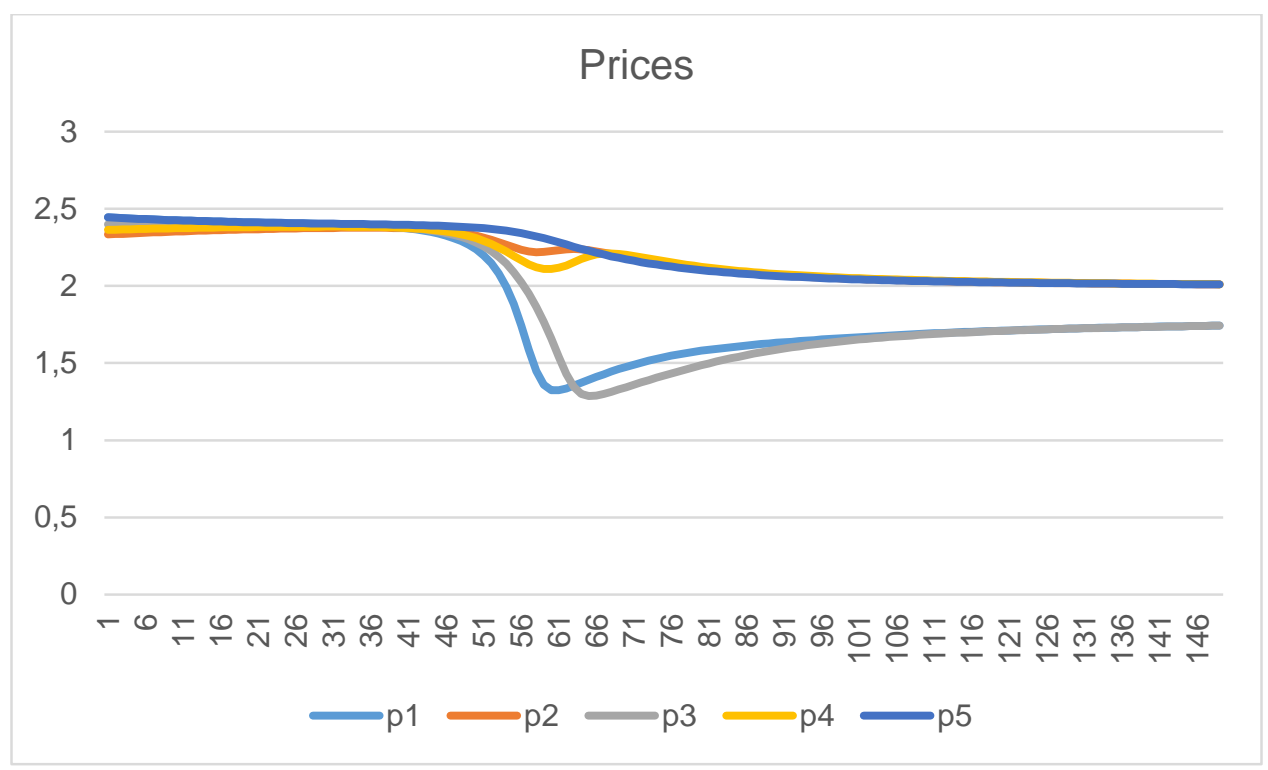

Figure 11. Simulation of (29) with five firms, prices, non-convergence to interior 


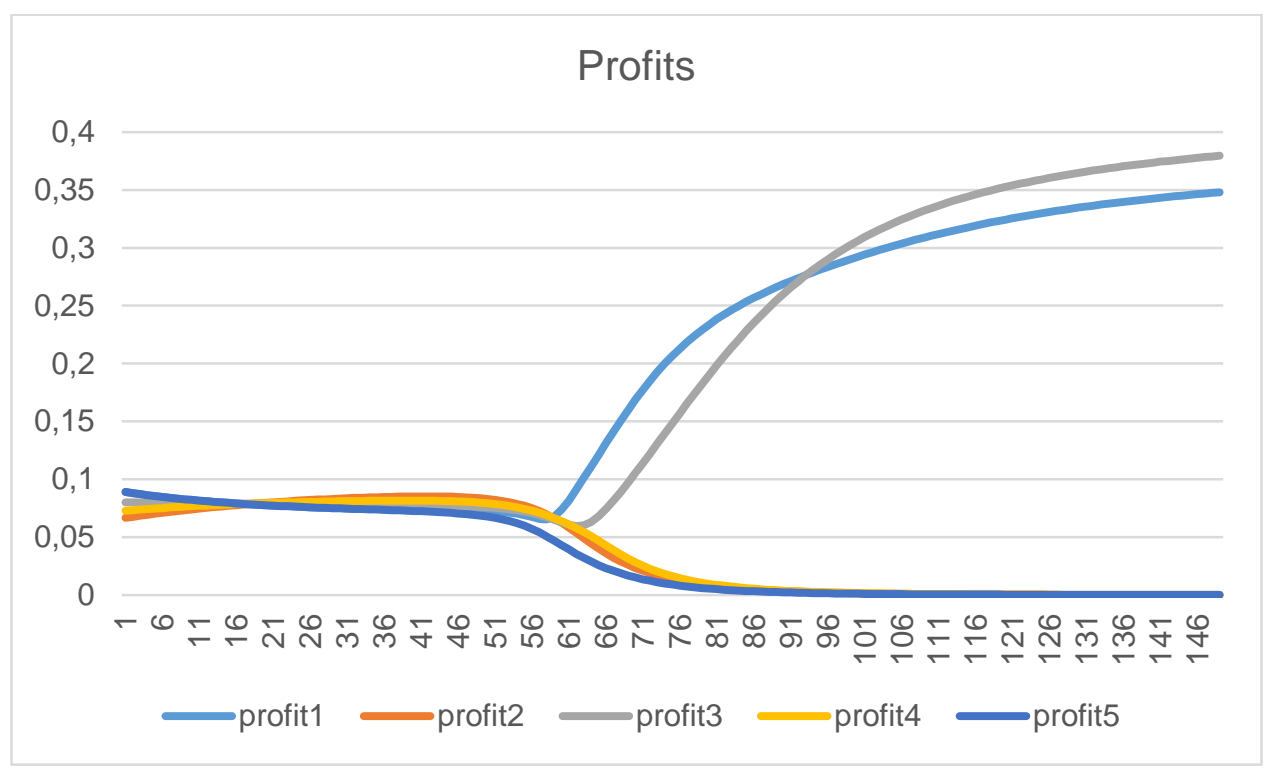

Figure 12. Simulation of (29) with five firms, profits, non-convergence to interior

Two price segments emerge in Figure 11 (which correspond to two different types of firm capabilities for technological advance, for the reduction of unit costs and for profits—see Figure 12), and the market ends up selecting (as we see in Figure 10) the most innovative firms 1 and 3. Still, notice that the surviving firms 1 and 3 are heterogeneous, both in their capacity to innovate and also in their respective mark-up parameters. We leave aside for future works the wide variety of experiments that can be carried out from this simple (but tractable and amenable to extensions and easy applications) evolutionary model, and we will now finish the paper by synthesizing our conclusions.

\section{Concluding remarks}

In this paper, we have devised and analyzed a tractable evolutionary model and, then, we have compared the closed-form results with the corresponding properties of a well-known neoclassical exemplar. We have also proposed an extension of the basic evolutionary setting in which we have considered cost heterogeneous firms and technological advance. We have seen that there is a great difference in the results, depending on whether we apply a fully-rational plus perfect-knowledge game, or an evolutionary dynamic process with bounded-rationality of the agents, imperfect information, learning, and ongoing change. 
As we have shown, when we assume bounded-rationality in the evolutionary model from both the point of view of firms (which do not maximize profits but are profit-seekers, and adjust their prices depending on demand change), and also from the point of view of consumers (who gradually learn better price options but still show myopia and inertial patterns), we obtain dynamics converging to a stable interior equilibrium where firms end up obtaining extra-profits and compete with moderate prices, and consumers do not see their welfare eroded by excessively high prices. These conclusions are essentially maintained in the extended version of the model. By contrast, within the neoclassical Bertrand game (with full rationality and perfect knowledge), at least in simple situations, firms do not obtain extra-profits (as they are incapable of maintaining a collusive agreement) and they end up competing over prices until they reach the null extra-profits Nash-state. Even in the case in which firms might collude in the repeated Bertrand setting, the resulting prices in collusion turn out to be higher than those emerging from the evolutionary model.

These different outcomes on market functioning lead us to pose the following reflection: the bounds on information in demand and supply, the dynamics of evolving market power, as well as the persistence of heterogeneity and the need to learn and adapt in evolutionary frameworks may bring about welfare patterns that may be more balanced than those arising in the Bertrand setting (with full-rationality and perfect-knowledge of the game). In the evolutionary case, prices and profits continuously adapt and change, the market tends to produce moderate prices and consumers do not have to bear such a high welfare cost as in the colluded situation of the Bertrand model. This comparative result is surprising, to say the least.

What we have found seems to indicate that the fact that the world is only partially understandable, that there are learning and adjustment costs, agents are different, and neither consumers nor firms act permanently as perfect-maximizers with complete knowledge, may not actually represent a series of market failures. On the contrary, these apparent imperfections may be at the very basis of what makes the market work. If this were the case, we should consider these aspects as key features of reality in order to improve our understanding of market processes. We believe that this is the line along which neoSchumpeterian evolutionary economics seeks to advance. The way ahead surely includes extending the analysis to confront price formation in multi-sector innovative co-evolutionary settings with price explanations in general equilibrium models. Likewise, comparative exercises consisting of testing neoclassical versus evolutionary results with real market data is clearly a very fruitful line for future empirical works. 


\section{Acknowledgements}

We would like to thank the participants at the $12^{\text {th }}$ Jena Summer Academy on Innovation and Uncertainty Jena 2018 for their comments and suggestions on previous versions of this work. We gratefully acknowledge the detailed comments by Bernardo Caldarola, Uwe Cantner, Martin von Kalthausen, Ekaterina Prytova, and Simone Vannuccini. We also want to thank for their very valuable comments to two anonymous referees of the Journal of Evolutionary Economics.

\section{References}

[1] Almudi, I., Fatas-Villafranca, F. and Izquierdo, L.R. (2012). Innovation, catch-up and leadership in science-based industries. Industrial and Corporate Change, 21: 345-375.

[2] Almudi, I., Fatas-Villafranca, F. and Izquierdo, L.R. (2013). Industry dynamics, technological regimes and the role of demand. Journal of Evolutionary Economics, 23: 1073-1098.

[3] Arrow, K. and Debreu, G. (1954). Existence of equilibrium for a competitive economy. Econometrica, 22: $265-290$.

[4] Arrow, K. and Hahn, F. (1971). General Competitive Analysis. Holden-Day. San Francisco.

[5] Bloch, H. and Metcalfe, J.S. (2018). Innovation, creative destruction and price theory. Industrial and Corporate Change, 27: 1-13.

[6] Debreu, G. (1959). Theory of Value. Yale University Press. New Haven.

[7] Dopfer, K. (ed). (2005). The Evolutionary Foundations of Economics. Cambridge University Press. New York.

[8] Dosi, G., Freeman, C., Nelson R., Silverberg G. and Soete L. (eds) (1988). Technical Change and Economic Theory. Pinter Publishers. London.

[9] Dosi, G., Grazzi, M., Marengo, L. and Settepanella, S. (2016). Production theory: accounting for firm heterogeneity and technological change. Journal of Industrial Economics, 64: 875-907.

[10] Dosi, G., Pereira, M. C., and Virgillito, M. E. (2017). The footprint of evolutionary processes of learning and selection upon the statistical properties of industrial dynamics. Industrial and Corporate Change, 26: 187-210.

[11] Fatas-Villafranca, F., Jarne G. and Sanchez-Choliz, J. (2009). Industrial leadership in science-based industries. A co-evolution model. Journal of Economic Behavior and Organization, 72: 390-407.

[12] Friedman, M. (1962). Price Theory. Aldine de Gruyter. New York. 
[13] Fudenberg, D. and Tirole, J. (1991). Game Theory. The MIT Press. Cambridge. MA.

[14] Gallegati, M., Di Guilmi, C. and Landini, S. (2017). Interactive Macroeconomics. Cambridge University Press. New York.

[15] Harsanyi, J and Selten, R. (1988). A General Theory of Equilibrium Selection in Games. The MIT Press. Cambridge, MA.

[16] Hart, S. and Mas-Colell, A. (2003). Uncoupled dynamics do not lead to Nash equilibrium. American Economic Review, 93: 1830-1836.

[17] Hayek, F. (1948). Individualism and Economic Order. University of Chicago Press. Chicago.

[18] Hofbauer, J. and Sigmund, K. (1998). Evolutionary Games and Population Dynamics. Cambridge University Press. Cambridge, UK.

[19] Kalecki, M. (1971). Selected Essays on the Dynamics of Capitalist Economies. Cambridge University Press. Cambridge, UK.

[20] Lucas, R.E. and Prescott, E.C. (1971). Investment under uncertainty. Econometrica, 39: 659-681. [21] Markey-Towler, B. (2016). Law of the jungle: firm survival and price dynamics in evolutionary markets. Journal of Evolutionary Economics, 26: 655-696.

[22] Metcalfe, J.S. (1998). Evolutionary Economics and Creative Destruction. Routledge. London.

[23] Nash, J. (1950). Equilibrium points in n-person games. Proceedings of the National Academy of Sciences, 36:46-49.

[24] Nelson, R.R. (2013). Demand, supply and their interaction on markets, as seen from the perspective of evolutionary economic theory. Journal of Evolutionary Economics, 23: 17-38.

[25] Nelson, R.R. (ed.) (2018). Modern Evolutionary Economics. Cambridge University Press. New York. [26] Nelson, R. R. and Winter, S. G. (1982). An Evolutionary Theory of Economic Change. Harvard University Press. Cambridge, MA.

[27] Polking, J. C. and Arnold, D. (2004). Ordinary Differential Equations Using MATLAB. Pearson/Prentice Hall. New York.

[28] Sandholm, W. H. (2010). Population Games and Evolutionary Dynamics. MIT Press. Cambridge, MA.

[29] Shubik, M. (1959). Strategy and Market Structure. Wiley. New York.

[30] Simon, H. A. (1965). Administrative Behavior: A study of decision-making processes in administrative organization. Free Press. New York.

[31] Stigler, G. J. (1966). The Theory of Price. ( $3^{\text {rd }}$ edition.) Macmillan. New York. 
[32] Stokey, N., Lucas, R. and Prescott, E. (1989). Recursive Methods in Economic Dynamics. Harvard University Press. Cambridge, MA.

[33] Tirole, J. (1988). The Theory of Industrial Organization. The MIT Press. Cambridge, MA.

[34] Van Damme, E. (1987). Stability and the Perfection of Nash Equilibria. Springer-Verlag. Berlin.

[35] Vives, X. (2001). Oligopoly Pricing. MIT Press. Cambridge, MA.

[36] Von Neumann, J. and Morgenstern, O. (1944). Theory of Games and Economic Behavior. Princeton University Press. Princeton.

[37] Weibull, J. W. (1995). Evolutionary Game Theory. The MIT Press. Cambridge, MA.

[38] Winter, S. G. (1971). Satisficing, selection and the innovating remnant. Quarterly Journal of Economics,85:57-84.

[39] Winter, S. G. (1984). Schumpeterian Competition in alternative technological regimes. Journal of Economic Behavior and Organization, 5: 287-320.

[40] Witt, U. (2001). Learning to consume: A theory of wants and the growth of demand. Journal of Evolutionary Economics, 11: 23-36.

[41] Witt, U. (2003). The Evolving Economy. Essays on the Evolutionary Approach to Economics. Edward Elgar. Cheltenham, UK. 